\title{
Sparse Bayesian Dictionary Learning with a Gaussian Hierarchical Model *
}

\author{
Linxiao Yang $^{\dagger} \quad$ Jun Fang ${ }^{\dagger} \quad$ Hong Cheng ${ }^{\ddagger} \quad$ Hongbin Li $^{\S}$
}

\begin{abstract}
We consider a dictionary learning problem aimed at designing a dictionary such that the signals admits a sparse or an approximate sparse representation over the learnt dictionary. The problem finds a variety of applications including image denoising, feature extraction, etc. In this paper, we propose a new hierarchical Bayesian model for dictionary learning, in which a Gaussian-inverse Gamma hierarchical prior is used to promote the sparsity of the representation. Suitable noninformative priors are also placed on the dictionary and the noise variance such that they can be reliably estimated from the data. Based on the hierarchical model, a variational Bayesian method and a Gibbs sampling method are developed for Bayesian inference. The proposed methods have the advantage that they do not require the knowledge of the noise variance a priori. Numerical results show that the proposed methods are able to learn the dictionary with an accuracy better than existing methods, particularly for the case where there is a limited number of training signals.

Keywords - Dictionary learning, Gaussian-inverse Gamma prior, variational Bayesian, Gibbs sampling.

\section{Introduction}

Sparse representation has been of significant interest over the past few years. It has found a variety of applications in practice as many natural signals admit a sparse or approximately sparse representation in a certain basis [1-3]. In many applications such as image denoising and interpolation, signals often have a sparse representation over a pre-specified non-adaptive dictionary, e.g. discrete cosine/wavelet transform (DCT/DWT) bases. Nevertheless, recent research $[4,5]$ has shown that

\footnotetext{
${ }^{*}$ This work was supported in part by the National Science Foundation of China under Grant 61172114, and the National Science Foundation under Grant ECCS-1408182.

${ }^{\dagger}$ Linxiao Yang, and Jun Fang (Corresponding Author) are with the National Key Laboratory on Com201321190224@std.uestc.edu.cn, JunFang@uestc.edu.cn

${ }^{\ddagger}$ Hong Cheng is with the School of Automation, University of Electronic Science and Technology of China, Chengdu 611731, China, Email: hcheng@uestc.edu.cn

${ }^{\S}$ Hongbin $\mathrm{Li}$ is with the Department of Electrical and Computer Engineering, Stevens Institute of Technology, Hoboken, NJ 07030, USA, E-mail: Hongbin.Li@stevens.edu
} munications, University of Electronic Science and Technology of China, Chengdu 611731, China, Emails:
\end{abstract}


the recovery, denoising and classification performance can be considerably improved by utilizing an adaptive dictionary that is learnt from training signals $[5,6]$. This has inspired studies on dictionary learning aimed to design overcompelete dictionaries that can better represent the signals. A number of algorithms, such as the K-singular value decomposition (K-SVD) [4], the method of optimal directions (MOD) [7], dictionary learning with the majorization method [8], and the simultaneous codeword optimization (SimCO) [9], were developed for overcomplete dictionary learning and sparse representation. Most algorithms formulate the dictionary learning as an optimization problem which is solved via a two-stage iterative process, namely, a sparse coding stage and a dictionary update stage. The main difference among these algorithms lies in the dictionary update stage. Specifically, the MOD method [7] updates the dictionary via solving a least square problem which admits a closed-form solution for dictionary update. The K-SVD algorithm [4], instead, updates the atoms of the dictionary in a sequential manner and while updating each atom, the atom is updated along with the nonzero entries in the corresponding row vector of the sparse matrix. The idea of sequential atom update was later extended to provide sequential update of multiple atoms each time [9], and recently generalized to parallel atom-updating in order to further accelerate the convergence of the iterative process [10]. These methods [4,7-10], although offering state-of-the-art performance, have several limitations. Specifically, they may require the knowledge of the sparsity level or the noise/residual variance for sparse coding (e.g. [4]), or this knowledge is needed for meticulously selecting some regularization parameters to properly control the tradeoff between the sparsity level and the data fitting error (e.g. $[8,10])$. In practice, however, the prior information about the noise variance and sparsity level is usually unavailable and an inaccurate estimation may result in substantial performance degradation. To mitigate these limitations, a nonparametric Bayesian dictionary learning method called beta-Bernoulli process factor analysis (BPFA) was recently developed in [11]. The proposed method can estimate the usage frequency of each atom, based on which the required number of atoms can be automatically inferred. Moreover, BPFA is also able to automatically infer the noise variance from the test image. These merits are deemed an important advantage over other dictionary learning methods. For [11], the posterior distributions cannot be derived analytically, and a Gibbs sampler was used for Bayesian inference. We also note that a class of online dictionary learning algorithms were developed in [12-16]. Unlike the above batch-based algorithms $[4,7,9,10]$ which use the whole set of training data for dictionary learning, online algorithms continuously update the dictionary using only one or a few (or a small amount of) training data, which enables them to handle very large data sets.

In this paper, we propose a new hierarchical Bayesian model for dictionary learning, in which a Gaussian-inverse Gamma hierarchical prior $[17,18]$ is used to promote the sparsity of the representation. Suitable non-informative priors are also placed on the dictionary and the noise variance 
such that they can be reliably inferred from the data. Based on the hierarchical model, a variational Bayesian method [19-21] and a Gibbs sampling method [22] are developed for Bayesian inference. For both inference methods, there are two different ways to update the dictionary: we can either update the whole set of atoms in one iteration, or update the atoms in a sequential manner. When updating the dictionary as a whole, the proposed variational Bayesian method has a dictionary update formula similar to the MOD method. For the Gibbs sampler, a sequential update seems able to expedite the convergence rate and helps achieve additional performance gain. Simulation results show that the proposed Gibbs sampling algorithm has notable advantages over other state-of-the-art dictionary learning methods in a number of interesting scenarios.

Note that the Gaussian-inverse Gamma hierarchical prior used in our paper is quite different from the beta-Bernoulli (also referred to as the spike-and-slab) prior employed in [11]. These two priors have their respective merits and both are widely used to promote the sparsity of solutions. In particular, the use of the Gaussian-inverse Gamma prior for sparse Bayesian learning has achieved great success in the framework of compressed sensing, e.g. [23-26]. It is therefore interesting to examine the problem of dictionary learning with such a prior and see if an additional performance improvement can be achieved. Note that the sparsity-promoting prior model (i.e. the hierarchical Gaussian-inverse Gamma prior) employed in this paper was also used in the sparse PCA framework (e.g. [27]). Nevertheless, to our best knowledge, our paper presents a first attempt to use the hierarchical Gaussian-inverse Gamma prior model to solve the dictionary learning problem. Although dictionary learning is closely related to sparse PCA [27], they still are two different problems with very distinct objectives: dictionary learning tries to learn an overcomplete dictionary to sparsely represent the observed data, whereas the sparse PCA aims to find a few sparse principle components of the underlying data matrix. Also, although sharing some degree of similarity, the prior model used in our paper is not exactly the same as the prior model in [27]. As a consequence, the derivations, update rules, and choice of model parameters in our work are different from those in [27]. Our work also provides an interesting comparison between two different inference methods, namely, the variational Bayes and the Gibbs sampling, for dictionary learning.

The rest of the paper is organized as follows. In Section 2, we introduce a hierarchical prior model for dictionary learning. Based on this hierarchical model, a variational Bayesian method and a Gibbs sampler are developed in Section 3 and Section 4 for Bayesian inference. Simulation results are provided in Section 5, followed by concluding remarks in Section 6 . 


\section{Hierarchical Model}

Suppose we have $L$ training signals $\left\{\boldsymbol{y}_{l}\right\}_{l=1}^{L}$, where $\boldsymbol{y}_{l} \in \mathbb{R}^{M}$. Dictionary learning aims at finding a common sparsifying dictionary $\boldsymbol{D} \in \mathbb{R}^{M \times N}$ such that these $L$ training signals admit a sparse representation over the overcomplete dictionary $\boldsymbol{D}$, i.e.

$$
\boldsymbol{y}_{l}=\boldsymbol{D} \boldsymbol{x}_{l}+\boldsymbol{w}_{l} \quad \forall l
$$

where $\boldsymbol{x}_{l}$ and $\boldsymbol{w}_{l}$ denote the sparse vector and the residual/noise vector, respectively. Define $\boldsymbol{Y} \triangleq$ $\left[\begin{array}{lll}\boldsymbol{y}_{1} & \ldots & \boldsymbol{y}_{L}\end{array}\right], \boldsymbol{X} \triangleq\left[\begin{array}{lll}\boldsymbol{x}_{1} & \ldots & \boldsymbol{x}_{L}\end{array}\right]$, and $\boldsymbol{W} \triangleq\left[\begin{array}{lll}\boldsymbol{w}_{1} & \ldots & \boldsymbol{w}_{L}\end{array}\right]$. The model (1) can be re-expressed as

$$
\boldsymbol{Y}=\boldsymbol{D} \boldsymbol{X}+\boldsymbol{W}
$$

Also, we write $\boldsymbol{D} \triangleq\left[\begin{array}{lll}\boldsymbol{d}_{1} & \ldots & \boldsymbol{d}_{N}\end{array}\right]$, where each column of the dictionary, $\boldsymbol{d}_{n}$, is called an atom.

In the following, we develop a Bayesian framework for learning the overcomplete dictionary and sparse vectors. To promote sparse representations, we assign a two-layer hierarchical Gaussianinverse Gamma prior to $\boldsymbol{X}$. The Gaussian-inverse Gamma prior is one of the most popular sparsitypromoting priors which has been widely used in compressed sensing [23,24,28]. Specifically, in the first layer, $\boldsymbol{X}$ is assigned a Gaussian prior distribution

$$
\begin{aligned}
p(\boldsymbol{X} \mid \boldsymbol{\alpha}) & =\prod_{n=1}^{N} \prod_{l=1}^{L} p\left(x_{n l}\right) \\
& =\prod_{n=1}^{N} \prod_{l=1}^{L} \mathcal{N}\left(x_{n l} \mid 0, \alpha_{n l}^{-1}\right),
\end{aligned}
$$

where $x_{n l}$ denotes the $(n, l)$ th entry of $\boldsymbol{X}$, and $\boldsymbol{\alpha} \triangleq\left\{\alpha_{n l}\right\}$ are non-negative sparsity-controlling hyperparameters. The notation $\mathcal{N}\left(x_{n l} \mid 0, \alpha_{n l}^{-1}\right)$ denotes Gaussian distribution with zero mean and variance $\alpha_{n l}^{-1}$. The second layer specifies Gamma distributions as hyperpriors over the hyperparameters $\left\{\alpha_{n l}\right\}$, i.e.

$$
\begin{aligned}
p(\boldsymbol{\alpha}) & =\prod_{n=1}^{N} \prod_{l=1}^{L} \operatorname{Gamma}\left(\alpha_{n l} ; a, b\right) \\
& =\prod_{n=1}^{N} \prod_{l=1}^{L} \Gamma(a)^{-1} b^{a} \alpha_{n l}^{a-1} e^{-b \alpha_{n l}},
\end{aligned}
$$

where $\Gamma(a)=\int_{0}^{\infty} t^{a-1} e^{-t} d t$ is the Gamma function. Here the notation Gamma $\left(\alpha_{n l} ; a, b\right)$ denotes the Gamma distribution of $\alpha_{n l}$ with parameter $a$ and $b$. To illustrate the sparsity-promoting property of the Gaussian-inverse Gamma prior, we integrate out the hyperparameter $\alpha_{n l}$ and obtain the marginal distribution of $x_{n l}$, which was shown to be a student-t distribution, i.e.

$$
\begin{aligned}
p\left(x_{n l}\right) & =\int p\left(x_{n l} \mid \alpha_{n l}\right) p\left(\alpha_{n} l ; a, b\right) d \alpha_{n l} \\
& =\frac{b^{a} \Gamma(a+0.5)}{(2 \pi)^{\frac{1}{2}} \Gamma(a)}\left(b+\frac{x_{n l}^{2}}{2}\right)^{-(a+0.5)} .
\end{aligned}
$$




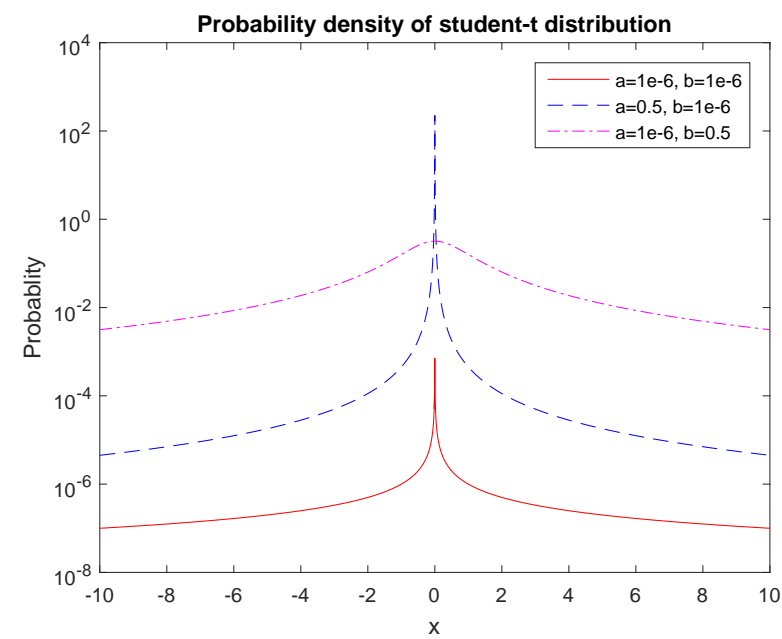

Figure 1: student-t distribution with different parameter setting

When $b$ is very small, say $b=10^{-6}$, the student-t distribution can be reduced to

$$
p\left(x_{n l}\right) \propto\left(\frac{1}{x_{n l}^{2}}\right)^{(a+0.5)} .
$$

We can easily see that (6) is a sparsity-promoting prior. Fig. 1 plots the student-t distributions with different choices of $a$ and $b$. We see that the distribution has a sharp peak around zero when $b$ is sufficiently small. Also, a larger $a$ results in a sharper peak, which implies that a larger $a$ leads to a more sparsity-encouraging prior. In our paper, the parameters $a$ and $b$ are chosen to be $a=0.5$ and $b=10^{-6}$.

In addition, in order to prevent the entries in the dictionary from becoming infinitely large, we assume the atoms of the dictionary $\left\{\boldsymbol{d}_{n}\right\}$ are mutually independent, and upon each atom we place a Gaussian prior, i.e.

$$
p(\boldsymbol{D})=\prod_{n=1}^{N} p\left(\boldsymbol{d}_{n}\right)=\prod_{n=1}^{N} \mathcal{N}\left(\boldsymbol{d}_{n} \mid \mathbf{0}, \beta \boldsymbol{I}\right),
$$

where $\beta$ is a parameter whose choice will be discussed later. The noise $\left\{\boldsymbol{w}_{l}\right\}$ are assumed independent multivariate Gaussian noise with zero mean and covariance matrix $(1 / \gamma) \boldsymbol{I}$, where the noise variance $1 / \gamma$ is assumed unknown a priori. To estimate the noise variance, we place a Gamma hyperprior over $\gamma$, i.e.

$$
p(\gamma)=\operatorname{Gamma}(\gamma ; c, d)=\Gamma(c)^{-1} d^{c} \gamma^{c-1} e^{-d \gamma},
$$

where we set $c=0.5$ and $d=10^{-6}$ to better suppress the data fitting error since a large $c$ encourages a large value of $\gamma$, i.e. a small noise variance. The proposed hierarchical model provides a general framework for learning the overcomplete dictionary, the sparse codes, as well as the noise variance. In the following, we develop a variational Beyesian method and a Gibbs sampling method for Bayesian inference. 


\section{Variational Inference}

\subsection{Review of The Variational Bayesian Methodology}

Before proceeding, we firstly provide a brief review of the variational Bayesian methodology. In a probabilistic model, let $\boldsymbol{y}$ and $\boldsymbol{\theta}$ denote the observed data and the hidden variables, respectively. It is straightforward to show that the marginal probability of the observed data can be decomposed into two terms [22]

$$
\ln p(\boldsymbol{y})=L(q)+\mathrm{KL}(q \| p),
$$

where

$$
L(q)=\int q(\boldsymbol{\theta}) \ln \frac{p(\boldsymbol{y}, \boldsymbol{\theta})}{q(\boldsymbol{\theta})} d \boldsymbol{\theta}
$$

and

$$
\mathrm{KL}(q \| p)=-\int q(\boldsymbol{\theta}) \ln \frac{p(\boldsymbol{\theta} \mid \boldsymbol{y})}{q(\boldsymbol{\theta})} d \boldsymbol{\theta}
$$

where $q(\boldsymbol{\theta})$ is any probability density function, $\operatorname{KL}(q \| p)$ is the Kullback-Leibler divergence [29] between $p(\boldsymbol{\theta} \mid \boldsymbol{y})$ and $q(\boldsymbol{\theta})$. Since $\mathrm{KL}(q \| p) \geq 0$, it follows that $L(q)$ is a rigorous lower bound for $\ln p(\boldsymbol{y})$. Moreover, notice that the left hand side of (9) is independent of $q(\boldsymbol{\theta})$. Therefore maximizing $L(q)$ is equivalent to minimizing $\operatorname{KL}(q \| p)$, and thus the posterior distribution $p(\boldsymbol{\theta} \mid \boldsymbol{y})$ can be approximated by $q(\boldsymbol{\theta})$ through maximizing $L(q)$.

The significance of the above transformation is that it circumvents the difficulty of computing the posterior probability $p(\boldsymbol{\theta} \mid \boldsymbol{y})$, when it is computationally intractable. For a suitable choice for the distribution $q(\boldsymbol{\theta})$, the quantity $L(q)$ may be more amiable to compute. Specifically, we could assume some specific parameterized functional form for $q(\boldsymbol{\theta})$ and then maximize $L(q)$ with respect to the parameters of the distribution. A particular form of $q(\boldsymbol{\theta})$ that has been widely used with great success is the factorized form over the component variables $\left\{\theta_{i}\right\}$ in $\boldsymbol{\theta}$ [19], i.e. $q(\boldsymbol{\theta})=\prod_{i} q_{i}\left(\theta_{i}\right)$. We therefore can compute the posterior distribution approximation by finding $q(\boldsymbol{\theta})$ of the factorized form that maximizes the lower bound $L(q)$. The maximization can be conducted in an alternating fashion for each latent variable, which leads to [19]

$$
q_{i}\left(\theta_{i}\right)=\frac{e^{\langle\ln p(\boldsymbol{y}, \boldsymbol{\theta})\rangle_{k \neq i}}}{\int e^{\langle\ln p(\boldsymbol{y}, \boldsymbol{\theta})\rangle_{k \neq i}} d \theta_{i}},
$$

where $\langle\cdot\rangle_{k \neq i}$ denotes the expectation with respect to the distributions $q_{k}\left(\theta_{k}\right)$ for all $k \neq i$. By taking the logarithm on the both side of (12), it can be equivalently written as

$$
\ln q_{i}\left(\theta_{i}\right)=\langle\ln p(\boldsymbol{y}, \boldsymbol{\theta})\rangle_{k \neq i}+\text { constant }
$$




\subsection{Proposed Variational Bayesian Method}

We now proceed to perform variational Bayesian inference for the proposed hierarchical model. Let $\boldsymbol{\theta} \triangleq\{\boldsymbol{X}, \boldsymbol{\alpha}, \boldsymbol{D}, \gamma\}$ denote all hidden variables. Our objective is to find the posterior distribution $p(\boldsymbol{\theta} \mid \boldsymbol{y})$. Since $p(\boldsymbol{\theta} \mid \boldsymbol{y})$ is usually computationally intractable, we, following the idea of [19], approximate $p(\boldsymbol{\theta} \mid \boldsymbol{y})$ by $q(\boldsymbol{X}, \boldsymbol{\alpha}, \boldsymbol{D}, \gamma)$ which has a factorized form over the hidden variables $\{\boldsymbol{X}, \boldsymbol{\alpha}, \boldsymbol{D}, \gamma\}$, i.e.

$$
q(\boldsymbol{X}, \boldsymbol{\alpha}, \boldsymbol{D}, \gamma)=q_{x}(\boldsymbol{X}) q_{\alpha}(\boldsymbol{\alpha}) q_{d}(\boldsymbol{D}) q_{\gamma}(\gamma)
$$

It is noted that such a factorized form leads to a compact estimate of $p(\boldsymbol{\theta} \mid \boldsymbol{y})$ with the correct mean [22]. We then maximize $L(q)$ defined in (10) with respect to $q_{x}(\boldsymbol{X}), q_{\alpha}(\boldsymbol{\alpha}), q_{d}(\boldsymbol{D})$, and $q_{\gamma}(\gamma)$, i.e.

$$
\max _{\left\{q_{x}(\boldsymbol{X}), q_{\alpha}(\boldsymbol{\alpha}), q_{d}(\boldsymbol{D}), q_{\gamma}(\gamma)\right\}} L(q) .
$$

As mentioned in the previous subsection, the maximization of $L(q)$ can be conducted in an alternating fashion for each latent variable, which leads to (details of the derivation can be found in [19])

$$
\begin{aligned}
\ln q_{x}(\boldsymbol{X}) & =\langle\ln p(\boldsymbol{Y}, \boldsymbol{X}, \boldsymbol{D}, \boldsymbol{\alpha}, \gamma)\rangle_{q_{d}(\boldsymbol{D}) q_{\alpha}(\boldsymbol{\alpha}) q_{\gamma}(\gamma)}+\mathrm{constant} \\
\ln q_{d}(\boldsymbol{D}) & =\langle\ln p(\boldsymbol{Y}, \boldsymbol{X}, \boldsymbol{D}, \boldsymbol{\alpha}, \gamma)\rangle_{q_{x}(\boldsymbol{X}) q_{\alpha}(\boldsymbol{\alpha}) q_{\gamma}(\gamma)}+\mathrm{constant} \\
\ln q_{\alpha}(\boldsymbol{\alpha}) & =\langle\ln p(\boldsymbol{Y}, \boldsymbol{X}, \boldsymbol{D}, \boldsymbol{\alpha}, \gamma)\rangle_{q_{x}(\boldsymbol{X}) q_{d}(\boldsymbol{D}) q_{\gamma}(\gamma)}+\mathrm{constant} \\
\ln q_{\gamma}(\gamma) & =\langle\ln p(\boldsymbol{Y}, \boldsymbol{X}, \boldsymbol{D}, \boldsymbol{\alpha}, \gamma)\rangle_{q_{x}(\boldsymbol{X}) q_{d}(\boldsymbol{D}) q_{\alpha}(\boldsymbol{\alpha})}+\mathrm{constant}
\end{aligned}
$$

where \langle\rangle$_{q_{1}(\cdot) \ldots q_{K}(\cdot)}$ denotes the expectation with respect to (w.r.t.) the distributions $\left\{q_{k}(\cdot)\right\}_{k=1}^{K}$. In summary, the posterior distribution approximations are computed in an alternating fashion for each hidden variable, with the distribution of other variables fixed. Details of this Bayesian inference scheme are provided next.

1). Update of $q_{x}(\boldsymbol{X})$ : The calculation of $q_{x}(\boldsymbol{X})$ can be decomposed into a set of independent tasks, with each task computing the posterior distribution approximation for each column of $\boldsymbol{X}$, i.e. $q_{x}\left(\boldsymbol{x}_{l}\right)$. We have

$$
\ln q_{x}\left(\boldsymbol{x}_{l}\right) \propto\left\langle\ln \left[p\left(\boldsymbol{y}_{l} \mid \boldsymbol{D}, \boldsymbol{x}_{l}, \gamma\right) p\left(\boldsymbol{x}_{l} \mid \boldsymbol{\alpha}_{l}\right)\right]\right\rangle_{q_{d}(\boldsymbol{D}) q_{\alpha}(\boldsymbol{\alpha}) q_{\gamma}(\gamma)}
$$

where $\boldsymbol{\alpha}_{l} \triangleq\left\{\alpha_{n l}\right\}_{n=1}^{N}$ are the sparsity-controlling hyperparameters associated with $\boldsymbol{x}_{l}, p\left(\boldsymbol{y}_{l} \mid \boldsymbol{D}, \boldsymbol{x}_{l}, \gamma\right)$ and $p\left(\boldsymbol{x}_{l} \mid \boldsymbol{\alpha}_{l}\right)$ are respectively given by

$$
\begin{aligned}
p\left(\boldsymbol{y}_{l} \mid \boldsymbol{D}, \boldsymbol{x}_{l}, \gamma\right) & =\left(\frac{\gamma}{2 \pi}\right)^{\frac{M}{2}} e^{-\frac{\gamma\left\|\boldsymbol{y}_{l}-\boldsymbol{D} \boldsymbol{x}_{l}\right\|_{2}^{2}}{2}} \\
p\left(\boldsymbol{x}_{l} \mid \boldsymbol{\alpha}_{l}\right) & =\prod_{n=1}^{N} \mathcal{N}\left(x_{n l} \mid 0, \alpha_{n l}^{-1}\right) .
\end{aligned}
$$


Substituting (17) into (16) and after some simplifications, it can be readily verified that $q_{x}\left(\boldsymbol{x}_{l}\right)$ follows a Gaussian distribution

$$
q_{x}\left(\boldsymbol{x}_{l}\right)=\mathcal{N}\left(\boldsymbol{x}_{l} \mid \boldsymbol{\mu}_{l}^{x}, \boldsymbol{\Sigma}_{l}^{x}\right)
$$

with its mean $\boldsymbol{\mu}_{l}^{x}$ and covariance matrix $\boldsymbol{\Sigma}_{l}^{x}$ given respectively as

$$
\begin{aligned}
& \boldsymbol{\mu}_{l}^{x}=\langle\gamma\rangle \boldsymbol{\Sigma}_{l}^{x}\langle\boldsymbol{D}\rangle^{T} \boldsymbol{y}_{l}, \\
& \boldsymbol{\Sigma}_{l}^{x}=\left(\langle\gamma\rangle\left\langle\boldsymbol{D}^{T} \boldsymbol{D}\right\rangle+\left\langle\boldsymbol{\Lambda}_{l}\right\rangle\right)^{-1},
\end{aligned}
$$

where $\langle\gamma\rangle$ denotes the expectation w.r.t. $q_{\gamma}(\gamma),\langle\boldsymbol{D}\rangle$ and $\left\langle\boldsymbol{D}^{T} \boldsymbol{D}\right\rangle$ denote the expectation w.r.t. $q_{d}(\boldsymbol{D})$, and $\left\langle\boldsymbol{\Lambda}_{l}\right\rangle \triangleq \operatorname{diag}\left(\left\langle\alpha_{1 l}\right\rangle, \ldots,\left\langle\alpha_{N l}\right\rangle\right)$, in which $\left\langle\alpha_{n l}\right\rangle$ represents the expectation w.r.t. $q_{\alpha}(\boldsymbol{\alpha})$. All these expectations are given by (30)-(35).

2). Update of $q_{d}(\boldsymbol{D})$ : The approximate posterior $q_{d}(\boldsymbol{D})$ can be obtained as

$$
\begin{aligned}
\ln q_{d}(\boldsymbol{D}) & \propto\langle\ln [p(\boldsymbol{Y} \mid \boldsymbol{X}, \boldsymbol{D}, \gamma) p(\boldsymbol{D})]\rangle_{q_{x}(\boldsymbol{X}) q_{\gamma}(\gamma)} \\
& \propto\left\langle-\gamma\|\boldsymbol{Y}-\boldsymbol{D} \boldsymbol{X}\|_{F}^{2}-\beta^{-1} \sum_{n=1}^{N} \boldsymbol{d}_{n}^{T} \boldsymbol{d}_{n}\right\rangle \\
& =\left\langle-\gamma \operatorname{tr}\left\{(\boldsymbol{Y}-\boldsymbol{D} \boldsymbol{X})(\boldsymbol{Y}-\boldsymbol{D} \boldsymbol{X})^{T}\right\}-\beta^{-1} \operatorname{tr}\left\{\boldsymbol{D} \boldsymbol{D}^{T}\right\}\right\rangle \\
& \propto\left\langle\operatorname{tr}\left\{\boldsymbol{D}\left(\gamma \boldsymbol{X} \boldsymbol{X}^{T}+\beta^{-1} \boldsymbol{I}\right) \boldsymbol{D}^{T}-2 \gamma \boldsymbol{Y} \boldsymbol{X}^{T} \boldsymbol{D}^{T}\right\}\right\rangle \\
& =\operatorname{tr}\left\{\boldsymbol{D}\left(\langle\gamma\rangle\left\langle\boldsymbol{X} \boldsymbol{X}^{T}\right\rangle+\beta^{-1} \boldsymbol{I}\right) \boldsymbol{D}^{T}-2\langle\gamma\rangle \boldsymbol{Y}\langle\boldsymbol{X}\rangle^{T} \boldsymbol{D}^{T}\right\},
\end{aligned}
$$

where for simplicity, we have dropped the subscripts of the $\langle\cdot\rangle$ operator. Define

$$
\begin{aligned}
& \boldsymbol{A} \triangleq\left(\langle\gamma\rangle\left\langle\boldsymbol{X} \boldsymbol{X}^{T}\right\rangle+\beta^{-1} \boldsymbol{I}\right)^{-1}, \\
& \boldsymbol{B} \triangleq\langle\gamma\rangle \boldsymbol{Y}\langle\boldsymbol{X}\rangle^{T},
\end{aligned}
$$

The posterior $q_{d}(\boldsymbol{D})$ can be further expressed as

$$
\begin{aligned}
\ln q_{d}(\boldsymbol{D}) & \propto \operatorname{tr}\left\{\boldsymbol{D} \boldsymbol{A}^{-1} \boldsymbol{D}^{T}-2 \boldsymbol{B} \boldsymbol{D}^{T}\right\} \\
& =\sum_{m=1}^{M}\left(\boldsymbol{d}_{m} \cdot \boldsymbol{A}^{-1} \boldsymbol{d}_{m}^{T}-2 \boldsymbol{b}_{m} \cdot \boldsymbol{d}_{m}^{T}\right),
\end{aligned}
$$

where $\boldsymbol{b}_{m}$. and $\boldsymbol{d}_{m}$. represents the $m$ th row of $\boldsymbol{B}$ and $\boldsymbol{D}$, respectively. It can be easily seen from (21) that the posterior distribution $q_{d}(\boldsymbol{D})$ has independent rows and each row follows a Gaussian distribution with its mean and covariance matrix given by $\boldsymbol{b}_{m} \cdot \boldsymbol{A}$ and $\boldsymbol{A}$, respectively, i.e.

$$
q_{d}(\boldsymbol{D})=\prod_{m=1}^{M} p\left(\boldsymbol{d}_{m} .\right)=\prod_{m=1}^{M} \mathcal{N}\left(\boldsymbol{b}_{m} . \boldsymbol{A}, \boldsymbol{A}\right) .
$$


3). Update of $q_{\alpha}(\boldsymbol{\alpha})$ : The variational optimization of $q_{\alpha}(\boldsymbol{\alpha})$ yields

$$
\begin{aligned}
\ln q_{\alpha}(\boldsymbol{\alpha}) & \propto\langle\ln p(\boldsymbol{X} \mid \boldsymbol{\alpha}) p(\boldsymbol{\alpha})\rangle_{q_{x}(\boldsymbol{X})} \\
& =\sum_{n=1}^{N} \sum_{l=1}^{L}\left\langle\ln p\left(x_{n l} \mid \alpha_{n l}\right) p\left(\alpha_{n l} ; a, b\right)\right\rangle \\
& \propto \sum_{n=1}^{N} \sum_{l=1}^{L}\left\{\left(a-\frac{1}{2}\right) \ln \alpha_{n l}-\left(b+\frac{\left\langle x_{n l}^{2}\right\rangle}{2}\right) \alpha_{n l}\right\} .
\end{aligned}
$$

Thus $q_{\alpha}(\boldsymbol{\alpha})$ has a form of a product of Gamma distributions

$$
q_{\alpha}(\boldsymbol{\alpha})=\prod_{n=1}^{N} \prod_{l=1}^{L} \operatorname{Gamma}\left(\alpha_{n l} ; \tilde{a}, \tilde{b}_{n l}\right),
$$

in which the parameters $\tilde{a}$ and $\tilde{b}_{n l}$ are respectively given as

$$
\tilde{a}=a+\frac{1}{2}, \quad \tilde{b}_{n l}=b+\frac{1}{2}\left\langle x_{n l}^{2}\right\rangle .
$$

4). Update of $q_{\gamma}(\gamma)$ : The variational optimization of $q_{\gamma}(\gamma)$ yields

$$
\begin{aligned}
\ln q_{\gamma}(\gamma) & \propto\langle\ln p(\boldsymbol{Y} \mid \boldsymbol{D}, \boldsymbol{X}, \gamma) p(\gamma)\rangle_{q_{d}(\boldsymbol{D}) q_{x}(\boldsymbol{X})} \\
\propto & \left\langle\ln \prod_{l=1}^{L} p\left(\boldsymbol{y}_{l} \mid \boldsymbol{D}, \boldsymbol{x}_{l}, \gamma\right) p(\gamma)\right\rangle \\
\propto & \left\langle\frac{M L}{2} \ln \gamma-\frac{\gamma}{2} \sum_{l=1}^{L}\left(\boldsymbol{y}_{l}-\boldsymbol{D} \boldsymbol{x}_{l}\right)^{T}\left(\boldsymbol{y}_{l}-\boldsymbol{D} \boldsymbol{x}_{l}\right)\right. \\
& +(c-1) \ln \gamma-d \gamma\rangle \\
= & \left(\frac{M L}{2}+c-1\right) \ln \gamma-\left(\frac{1}{2}\left\langle\|\boldsymbol{Y}-\boldsymbol{D} \boldsymbol{X}\|_{F}^{2}\right\rangle+d\right) \gamma
\end{aligned}
$$

Therefore $q_{\gamma}(\gamma)$ follows a Gamma distribution

$$
q_{\gamma}(\gamma)=\operatorname{Gamma}(\gamma \mid \tilde{c}, \tilde{d})
$$

with the parameters $\tilde{c}$ and $\tilde{d}$ given respectively by

$$
\begin{aligned}
& \tilde{c}=\frac{M L}{2}+c, \\
& \tilde{d}=d+\frac{1}{2}\left\langle\|\boldsymbol{Y}-\boldsymbol{D} \boldsymbol{X}\|_{F}^{2},\right\rangle
\end{aligned}
$$

where

$$
\begin{aligned}
\left\langle\|\boldsymbol{Y}-\boldsymbol{D} \boldsymbol{X}\|_{F}^{2}\right\rangle= & \left\langle\operatorname{tr}\left\{(\boldsymbol{Y}-\boldsymbol{D} \boldsymbol{X})^{T}(\boldsymbol{Y}-\boldsymbol{D} \boldsymbol{X})\right\}\right\rangle \\
= & \|\boldsymbol{Y}-\langle\boldsymbol{D}\rangle\langle\boldsymbol{X}\rangle\|_{F}^{2}+\operatorname{tr}\left\{\left\langle\boldsymbol{D}^{T} \boldsymbol{D}\right\rangle\left\langle\boldsymbol{X} \boldsymbol{X}^{T}\right\rangle\right\} \\
& -\operatorname{tr}\left\{\left\langle\boldsymbol{D}^{T}\right\rangle\langle\boldsymbol{D}\rangle\langle\boldsymbol{X}\rangle\left\langle\boldsymbol{X}^{T}\right\rangle\right\} .
\end{aligned}
$$


In summary, the variational Bayesian inference involves updates of the approximate posterior distributions for hidden variables $\boldsymbol{X}, \boldsymbol{D}, \boldsymbol{\alpha}$, and $\gamma$. Some of the expectations and moments used during the update are summarized as

$$
\begin{aligned}
\left\langle x_{n l}^{2}\right\rangle & =\left(\boldsymbol{\mu}_{l}^{x}[n]\right)^{2}+\boldsymbol{\Sigma}_{l}^{x}[n, n], \\
\left\langle\boldsymbol{X} \boldsymbol{X}^{T}\right\rangle & =\langle\boldsymbol{X}\rangle\langle\boldsymbol{X}\rangle^{T}+\sum_{l=1}^{L} \boldsymbol{\Sigma}_{l}^{x}, \\
\langle\boldsymbol{D}\rangle & =\boldsymbol{B} \boldsymbol{A}, \\
\left\langle\boldsymbol{D}^{T} \boldsymbol{D}\right\rangle & =\langle\boldsymbol{D}\rangle^{T}\langle\boldsymbol{D}\rangle+M\langle\boldsymbol{A}\rangle, \\
\left\langle\alpha_{n l}\right\rangle & =\tilde{a} / \tilde{b}_{n l}, \\
\langle\gamma\rangle & =\tilde{c} / \tilde{d} .
\end{aligned}
$$

where in (30), $\boldsymbol{\mu}_{l}^{x}[n]$ denotes the $n$th entry of $\boldsymbol{\mu}_{l}^{x}, \boldsymbol{\Sigma}_{l}^{x}[n, n]$ represents the $n$th diagonal element of $\boldsymbol{\Sigma}_{l}^{x}$, and (32) follows from (22). So far we have developed a variational Bayesian method for approximating the posterior. After the algorithm converges, all the variables can be estimated by maximizing the approximated posterior. Since both $q_{d}(\boldsymbol{D})$ and $q_{x}(\boldsymbol{X})$ follow the Gaussian distribution, the estimate of $\boldsymbol{D}$ and $\boldsymbol{X}$ are given by $\langle\boldsymbol{D}\rangle$ and $\langle\boldsymbol{X}\rangle$. For simplicity, we set $\langle\boldsymbol{\alpha}\rangle$ and $\langle\gamma\rangle$ as the estimate of $\boldsymbol{\alpha}$ and $\gamma$, respectively. For clarity, we summarize our algorithm as Algorithm 1.

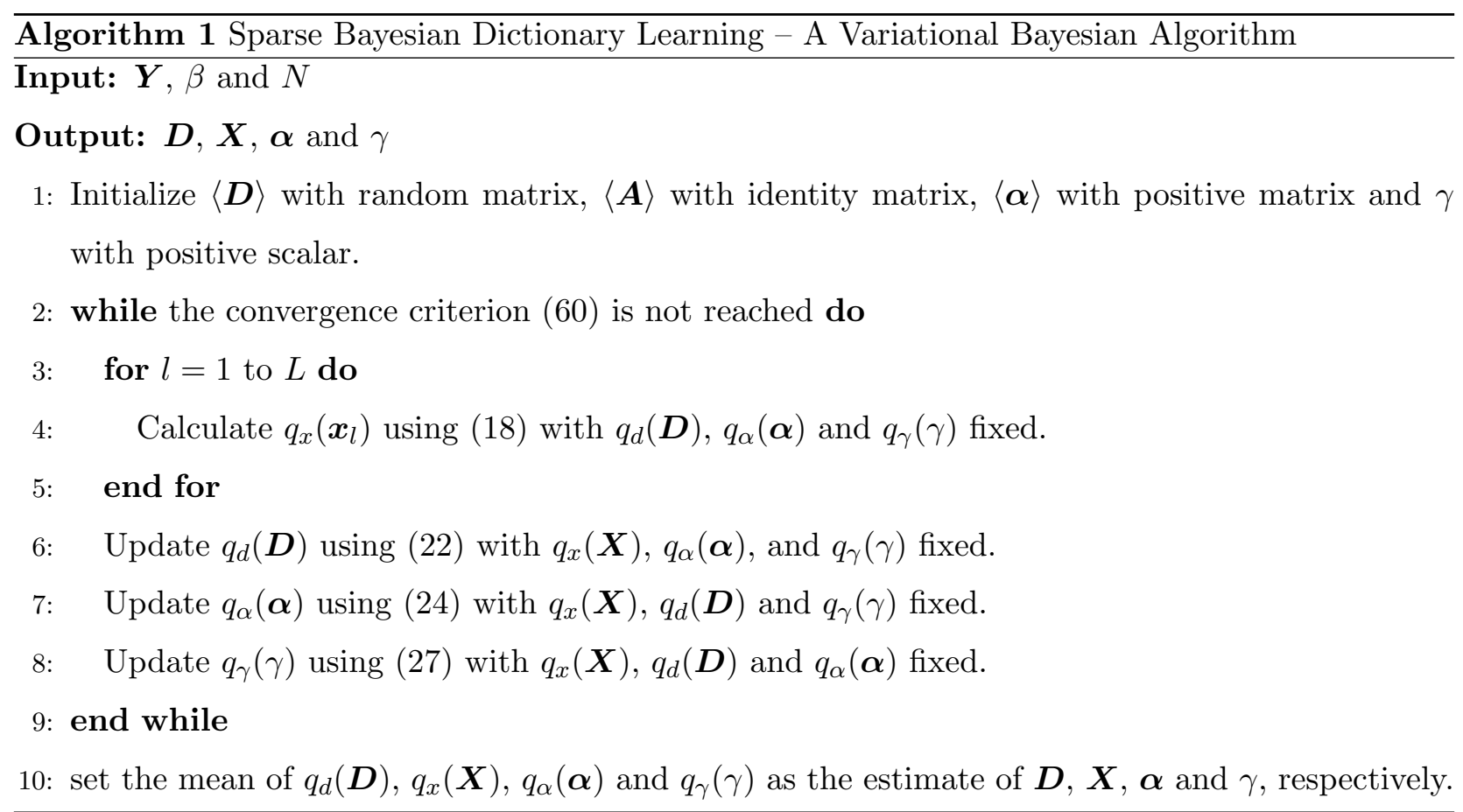

Remarks 1: We discuss the choice of the parameter $\beta$ which defines the variance of the dictionary atoms. We might like to set $\beta$ equal to $1 / m$ such that the norm of each atom has unit variance. Our experiment results, however, suggest that a very large value of $\beta$, e.g. $10^{8}$, leads to better 
performance. In fact, choosing an infinitely large $\beta$ implies placing strictly non-informative priors over the atoms $\left\{\boldsymbol{d}_{n}\right\}$, in which case the update of the dictionary is simplified as

$$
\langle\boldsymbol{D}\rangle=\boldsymbol{B} \boldsymbol{A}=\boldsymbol{Y}\left\langle\boldsymbol{X}^{T}\right\rangle\left\langle\boldsymbol{X} \boldsymbol{X}^{T}\right\rangle^{-1} .
$$

This update formula is similar to the formula used for dictionary update in the MOD method, except that the point estimates $\boldsymbol{X}$ and $\boldsymbol{X} \boldsymbol{X}^{T}$ are now replaced by the posterior mean $\langle\boldsymbol{X}\rangle$ and $\left\langle\boldsymbol{X} \boldsymbol{X}^{T}\right\rangle$, respectively.

In the above algorithm, atoms are updated in a parallel way. By assuming posterior independence among atoms $\left\{\boldsymbol{d}_{n}\right\}$, our method can also be readily adapted to provide sequential update of the atoms, i.e. it updates one atom at a time while fixing the rest atoms in the dictionary. The mean field approximation, in this case, can be expressed as

$$
\begin{aligned}
p(\boldsymbol{\theta} \mid \boldsymbol{y}) & \approx q(\boldsymbol{x}, \boldsymbol{\alpha}, \boldsymbol{D}, \gamma) \\
& =q_{x}(\boldsymbol{x}) q_{\alpha}(\boldsymbol{\alpha}) \prod_{n=1}^{N} q_{d_{n}}\left(\boldsymbol{d}_{n}\right) q_{\gamma}(\gamma) .
\end{aligned}
$$

The posterior distribution $q_{d_{n}}\left(\boldsymbol{d}_{n}\right)$ can then be computed by maximizing $L(q)$ while keeping the distributions of other hidden variables fixed, which leads to

$$
\begin{aligned}
& \ln q_{d_{n}}\left(\boldsymbol{d}_{n}\right) \\
\propto & \left\langle\ln p\left(\boldsymbol{Y}, \boldsymbol{X},\left\{\boldsymbol{d}_{k}\right\}, \boldsymbol{\alpha}, \gamma\right)\right\rangle_{q_{x}(\boldsymbol{X}) \prod_{k \neq n}^{N} q_{d_{k}}\left(\boldsymbol{d}_{k}\right) q_{\alpha}(\boldsymbol{\alpha}) q_{\gamma}(\gamma)} \\
\propto & \left\langle\ln p\left(\boldsymbol{Y} \mid \boldsymbol{X},\left\{\boldsymbol{d}_{k}\right\}, \gamma\right) p\left(\boldsymbol{d}_{n}\right)\right\rangle_{q_{x}(\boldsymbol{X}) \prod_{k \neq n}^{N} q_{d_{k}}\left(\boldsymbol{d}_{k}\right) q_{\gamma}(\gamma)} \\
\stackrel{(a)}{\propto}\left\langle\ln p\left(\boldsymbol{Y}^{-n} \mid \boldsymbol{d}_{n}, \boldsymbol{x}_{n .}, \gamma\right) p\left(\boldsymbol{d}_{n}\right)\right\rangle_{q_{x}(\boldsymbol{X}) \prod_{k \neq n}^{N} q_{d_{k}}\left(\boldsymbol{d}_{k}\right) q_{\gamma}(\gamma)} & (b) \\
\propto & \frac{1}{2}\left\langle\gamma \operatorname{tr}\left\{\left(\boldsymbol{Y}^{-n}-\boldsymbol{d}_{n} \boldsymbol{x}_{n .}\right)\left(\boldsymbol{Y}^{-n}-\boldsymbol{d}_{n} \boldsymbol{x}_{n .}\right)^{T}\right\}\right. \\
& \left.+\beta^{-1} \boldsymbol{d}_{n}^{T} \boldsymbol{d}_{n}\right\rangle \\
= & \frac{1}{2}\left[\boldsymbol{d}_{n}^{T}\left(\langle\gamma\rangle\left\langle\boldsymbol{x}_{n} . \boldsymbol{x}_{n .}^{T}\right\rangle+\beta^{-1}\right)^{-1} \boldsymbol{d}_{n}-2 \boldsymbol{d}_{n}\left\langle\boldsymbol{Y}^{-n}\right\rangle\left\langle\boldsymbol{x}_{n .}^{T} .\right\rangle\right]
\end{aligned}
$$

where in $(a)$, we define

$$
\boldsymbol{Y}^{-n} \triangleq \boldsymbol{Y}-\boldsymbol{D}^{-n} \boldsymbol{X}
$$

in which $\boldsymbol{D}^{-n}$ is generated by $\boldsymbol{D}$ with the $n$th column of $\boldsymbol{D}$ replaced by a zero vector, and $\boldsymbol{x}_{n}$. denotes the $n$th row of $\boldsymbol{X},(b)$ comes from the fact that $\boldsymbol{Y}^{-n}-\boldsymbol{d}_{n} \boldsymbol{x}_{n}=\boldsymbol{W}$ and thus we have

$$
p\left(\boldsymbol{Y}^{-n} \mid \boldsymbol{d}_{n}, \boldsymbol{x}_{n \cdot}, \gamma\right)=\frac{\gamma^{\frac{M L}{2}}}{2 \pi} \exp \left(-\frac{1}{2} \gamma \| \boldsymbol{Y}^{-n}-\boldsymbol{d}_{n} \boldsymbol{x}_{n \cdot \|_{F}^{2}}\right) .
$$

From (38), it can be seen that $\boldsymbol{d}_{n}$ follows a Gaussian distribution

$$
q_{d_{n}}\left(\boldsymbol{d}_{n}\right)=\mathcal{N}\left(\boldsymbol{d}_{n} \mid \boldsymbol{\mu}_{n}^{d}, \boldsymbol{\Sigma}_{n}^{d}\right),
$$


with the mean and the covariance matrix given respectively by

$$
\begin{aligned}
\boldsymbol{\mu}_{n}^{d} & =\boldsymbol{\Sigma}_{n}^{d}\left\langle\boldsymbol{Y}^{-n}\right\rangle\left\langle\boldsymbol{x}_{n .}^{T} .\right\rangle, \\
\boldsymbol{\Sigma}_{n}^{d} & =\left(\langle\gamma\rangle\left\langle\boldsymbol{x}_{n} . \boldsymbol{x}_{n .}^{T}\right\rangle+\beta^{-1}\right)^{-1} \boldsymbol{I},
\end{aligned}
$$

where $\left\langle\boldsymbol{x}_{n} . \boldsymbol{x}_{n}^{T}\right.$. $\rangle$ is the $n$th diagonal element of $\left\langle\boldsymbol{X} \boldsymbol{X}^{T}\right\rangle$, and $\left\langle\boldsymbol{Y}^{-n}\right\rangle=\boldsymbol{Y}-\left\langle\boldsymbol{D}^{-n}\right\rangle\langle\boldsymbol{X}\rangle$. Our proposed algorithm therefore can be readily extended to a columnwise update procedure by replacing the update of $q_{d}(\boldsymbol{D})$ with the sequential update of $q_{d_{n}}\left(\boldsymbol{d}_{n}\right), \forall n$. We note that since our experimental results show that the sequential update of $q_{d_{n}}\left(\boldsymbol{d}_{n}\right), \forall n$ suffers from the numerical instability, it is not put in the experiments.

\section{Gibbs Sampler}

Gibbs sampling is an alternative to the variational Bayes method for Bayesian inference. In particular, different from the variational Bayes which provides a locally-optimal, exact analytical solution to an approximation of the posterior, Monte Carlo techniques such as Gibbs sampling provide a numerical approximation to the exact posterior of hidden variables using a set of samples. It has been observed that the Gibbs sampler may provide better performance than the variational Bayesian inference in some cases [30].

Let $\boldsymbol{\theta} \triangleq\{\boldsymbol{X}, \boldsymbol{\alpha}, \boldsymbol{D}, \gamma\}$ denote all hidden variables in our hierarchical model. We aim to find the posterior distribution of $\boldsymbol{\theta}$ given the observed data $\boldsymbol{Y}$

$$
p(\boldsymbol{\theta} \mid \boldsymbol{Y}) \propto p(\boldsymbol{Y} \mid \boldsymbol{D}, \boldsymbol{X}, \gamma) p(\boldsymbol{D}) p(\boldsymbol{X} \mid \boldsymbol{\alpha}) p(\boldsymbol{\alpha}) p(\gamma) .
$$

To provide an approximation to the posterior distribution of the hidden variables, the Gibbs sampler generates an instance from the distribution of each hidden variable in turn, conditional on the current values of the other hidden variables. It can be shown (see, for example, [31]) that the sequence of samples constitutes a Markov chain, and the stationary distribution of that Markov chain is just the sought-after joint distribution. Specifically, the sequential sampling procedure of the Gibbs sampler is given as follows.

- Sampling $\boldsymbol{X}$ according to its conditional marginal distribution $p\left(\boldsymbol{X} \mid \boldsymbol{Y}, \boldsymbol{D}^{(t)}, \boldsymbol{\alpha}^{(t)}, \gamma^{(t)}\right)$;

- Sampling $\boldsymbol{D}$ according to its conditional marginal distribution $p\left(\boldsymbol{D} \mid \boldsymbol{Y}, \boldsymbol{X}^{(t+1)}, \boldsymbol{\alpha}^{(t)}, \gamma^{(t)}\right)$;

- Sampling $\boldsymbol{\alpha}$ according to its conditional marginal distribution $p\left(\boldsymbol{\alpha} \mid \boldsymbol{Y}, \boldsymbol{D}^{(t+1)}, \boldsymbol{X}^{(t+1)}, \gamma^{(t)}\right)$;

- Sampling $\gamma$ according to its conditional marginal distribution $p\left(\gamma \mid \boldsymbol{Y}, \boldsymbol{D}^{(t+1)}, \boldsymbol{X}^{(t+1)}, \boldsymbol{\alpha}^{(t+1)}\right)$. 
Note that the above sampling scheme is also referred to as a blocked Gibbs sampler [22] because it groups two or more variables together and samples from their joint distribution conditioned on all other variables, rather than sampling from each one individually. Details of this sampling scheme are provided next. For simplicity, the notation $p(\boldsymbol{z} \mid-)$ is used in the following to denote the distribution of variable $\boldsymbol{z}$ conditioned on all other variables.

1). Sampling $\boldsymbol{X}$ : Samples of $\boldsymbol{X}$ can be obtained by independently sampling each column of $\boldsymbol{X}$, i.e. $\boldsymbol{x}_{l}$. The conditional marginal distribution of $\boldsymbol{x}_{l}$ is given as

$$
\begin{aligned}
p\left(\boldsymbol{x}_{l} \mid-\right) & \propto p(\boldsymbol{Y} \mid \boldsymbol{X}, \boldsymbol{D}, \gamma) p\left(\boldsymbol{x}_{l} \mid \boldsymbol{\alpha}_{l}\right) \\
& \propto p\left(\boldsymbol{y}_{l} \mid \boldsymbol{D}, \boldsymbol{x}_{l}, \gamma\right) p\left(\boldsymbol{x}_{l} \mid \boldsymbol{\alpha}_{l}\right) .
\end{aligned}
$$

Recalling (17), it can be easily verified that $p\left(\boldsymbol{x}_{l} \mid-\right)$ follows a Gaussian distribution

$$
p\left(\boldsymbol{x}_{l} \mid-\right)=\mathcal{N}\left(\boldsymbol{\mu}_{l}^{x}, \boldsymbol{\Sigma}_{l}^{x}\right)
$$

with its mean $\boldsymbol{\mu}_{l}^{x}$ and covariance matrix $\boldsymbol{\Sigma}_{l}^{x}$ given by

$$
\begin{aligned}
& \boldsymbol{\mu}_{l}^{x}=\gamma \boldsymbol{\Sigma}_{l}^{x} \boldsymbol{D}^{T} \boldsymbol{y}_{l}, \\
& \boldsymbol{\Sigma}_{l}^{x}=\left(\gamma \boldsymbol{D}^{T} \boldsymbol{D}+\boldsymbol{\Lambda}_{l}\right)^{-1},
\end{aligned}
$$

where $\boldsymbol{\Lambda}_{l} \triangleq \operatorname{diag}\left(\alpha_{1 l}, \ldots, \alpha_{N l}\right)$.

2). Sampling D: There are two different ways to sample the dictionary: we can sample the whole set of atoms simultaneously, or sample the atoms in a successive way. We note that the successive sample way is likes a Gauss-Seidel algorithms while directly sample the whole dictionary which is essentially a parallel sample way is likes the Jacobi algorithms. Thus we can expect that sampling the atoms of the dictionary in a sequential manner converges fast than sampling the whole atoms simultaneously. Here, in order to expedite the convergence of the Gibbs sampler, we sample the atoms of the dictionary in a sequential manner. The conditional distribution of $\boldsymbol{d}_{n}$ can be written as

$$
\begin{aligned}
p\left(\boldsymbol{d}_{n} \mid-\right) & \propto p\left(\boldsymbol{d}_{n}\right) p(\boldsymbol{Y} \mid \boldsymbol{D}, \boldsymbol{X}, \gamma) \\
& \propto p\left(\boldsymbol{d}_{n}\right) p\left(\boldsymbol{Y}^{-n} \mid \boldsymbol{d}_{n}, \boldsymbol{x}_{n .}, \gamma\right),
\end{aligned}
$$

where $\boldsymbol{Y}^{-n}$ is defined in (39). Recalling (40), we can show that the conditional distribution of $\boldsymbol{d}_{n}$ follows a Gaussian distribution

$$
p\left(\boldsymbol{d}_{n} \mid-\right)=\mathcal{N}\left(\boldsymbol{\mu}_{n}^{d}, \boldsymbol{\Sigma}_{n}^{d}\right),
$$

with its mean and covariance matrix given by

$$
\begin{aligned}
\boldsymbol{\mu}_{n}^{d} & =\gamma \boldsymbol{\Sigma}_{n}^{d} \boldsymbol{Y}^{-n} \boldsymbol{x}_{n .}^{T}, \\
\boldsymbol{\Sigma}_{n}^{d} & =\left(\gamma \boldsymbol{x}_{n} \cdot \boldsymbol{x}_{n}^{T}+\beta^{-1}\right)^{-1} \boldsymbol{I} .
\end{aligned}
$$


3). Sampling $\boldsymbol{\alpha}$ : The log-conditional distribution of $\alpha_{n l}$ can be computed as

$$
\begin{aligned}
\ln p\left(\alpha_{n l} \mid-\right) & \propto \ln p\left(\alpha_{n l} ; a, b\right) p\left(x_{n l} \mid \alpha_{n l}\right) \\
& \propto\left(a-\frac{1}{2}\right) \ln \alpha_{n l}-\left(b+\frac{x_{n l}^{2}}{2}\right) .
\end{aligned}
$$

It is easy to verify that $\alpha_{n l}$ still follows a Gamma distribution

$$
p\left(\alpha_{n l} \mid-\right)=\operatorname{Gamma}\left(\hat{a}, \hat{b}_{n l}\right)
$$

with the parameters $\hat{a}$ and $\hat{b}_{n l}$ given as

$$
\begin{gathered}
\hat{a}=a+\frac{1}{2}, \\
\hat{b}_{n l}=b+\frac{1}{2} x_{n l}^{2} .
\end{gathered}
$$

4). Sampling $\gamma$ : The log-conditional distribution of $\gamma$ is given by

$$
\begin{aligned}
\ln p(\gamma \mid-) & \propto \ln p(\boldsymbol{Y} \mid \boldsymbol{D}, \boldsymbol{X}, \gamma) p(\gamma) \\
& \propto \ln \prod_{l=1}^{L} p\left(\boldsymbol{y}_{l} \mid \boldsymbol{D}, \boldsymbol{x}_{l}, \gamma\right) p(\gamma) \\
& =\left(\frac{M L}{2}+c-1\right) \ln \gamma-\left(\frac{1}{2}\|\boldsymbol{Y}-\boldsymbol{D} \boldsymbol{X}\|_{F}^{2}+d\right) \gamma
\end{aligned}
$$

from which we can arrive at

$$
p(\gamma \mid-)=\operatorname{Gamma}(\hat{c}, \hat{d})
$$

where

$$
\begin{aligned}
& \hat{c}=a+\frac{M L}{2}, \\
& \hat{d}=d+\frac{1}{2}\|\boldsymbol{Y}-\boldsymbol{D} \boldsymbol{X}\|_{F}^{2} .
\end{aligned}
$$

So far we have derived the conditional marginal distributions for hidden variables $\{\boldsymbol{D}, \boldsymbol{X}, \boldsymbol{\alpha}, \gamma\}$. Gibbs sampler successively generates the samples of these variables according to their conditional distributions. After a burn-in period [32], the generated samples can be viewed as samples drawn from the posterior distribution $p(\boldsymbol{X}, \boldsymbol{D}, \boldsymbol{\alpha}, \gamma \mid \boldsymbol{Y})$. With those samples, all the variables can be estimated by averaging the last few samples of the Gibbs sampler. For clarity, we now summarize the Gibbs sampling algorithm as Algorithm 2.

\section{$5 \quad$ Simulation Results}

We now carry out experiments to illustrate the performance of our proposed sparse Bayesian dictionary learning (SBDL) methods, which are respectively referred to as SBDL-VB and SBDL-Gibbs. 


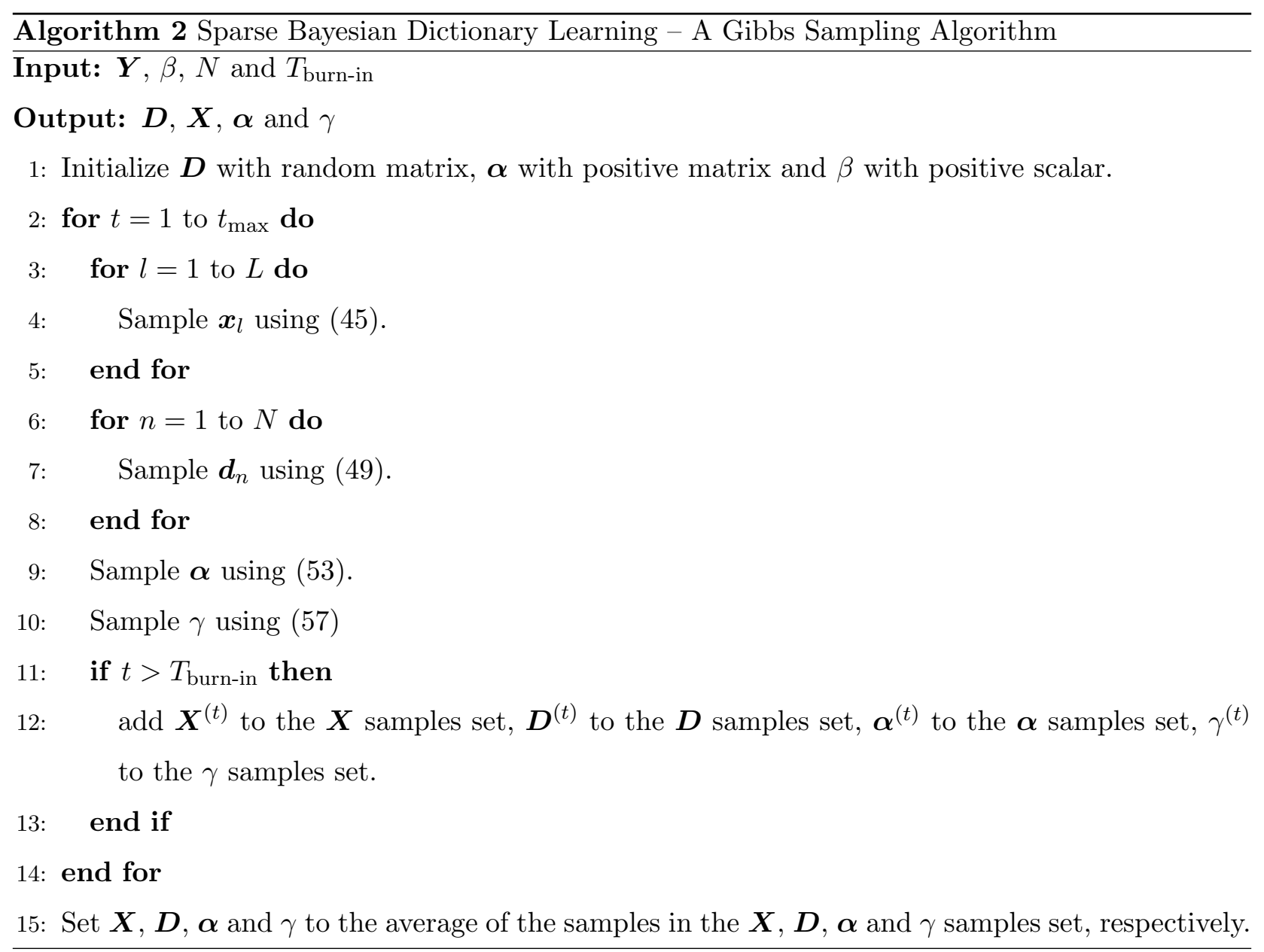


Throughout our experiments, the parameters for our proposed method are set equal to $a=0.5$, $b=10^{-6}, c=0.5$ and $d=10^{-6}$. The parameter $\beta$ is set to $\beta=10^{8}$ for the SBDL-VB. Note that the SBDL-Gibbs is insensitive to the choice $\beta$ and here we simply choose $\beta=1$. Due to the presence of noise, the sparse coding $\boldsymbol{X}$ may finally converge to an approximately sparse solution which contains many small values and an absolutely sparse $\boldsymbol{X}$ requires a pruning operation. Due to its simplicity and fast execution, the orthogonal matching pursuit (OMP) method is employed to perform the sparse coding in all of our experiments for SBDL-VB and SBDL-Gibbs after the dictionary learning. The termination condition of OMP is set to such that the $\ell_{2}$ norm of measurement residual smaller than $C \sigma \sqrt{M}$, where $\sigma$ is the noise standard deviation, $M$ is length of samples and $C$ is a factor. We set $C$ to 1 for synthetic data and 1.15 to image denoising as suggested in [5]. We compare our proposed methods with other several existing state-of-the-art dictionary learning methods, namely, the K-SVD algorithm [4], the atom parallel-updating (APrU-DL) method [10], and the Bata-Bernoulli process factor analysis (BPFA) method [11]. Both synthetic data and real data are used to test the performance of respective algorithms. For SBDL-VB, we continue the iterative process until the difference between the estimated dictionary of successive iterations is negligible, i.e.

$$
\frac{\left\|\boldsymbol{D}^{(t+1)}-\boldsymbol{D}^{(t)}\right\|_{F}}{\|\boldsymbol{Y}\|_{F}}<10^{-3}
$$

For SBDL-Gibbs, the number of iterations is set to 300 , i.e. $t_{\max }=300$ and the estimate of the dictionary is simply chosen to be the last sample of the Gibbs sampler, which implies that $T_{\text {burn-in }}=299$. Our experimental results shows that 300 iterations is enough for the Gibbs sampler to achieve a decent result. For a fair comparison, the competing algorithms including K-SVD, APrUDL, and BPFA are executed with sufficient numbers of iterations to achieve their best performance.

\subsection{Synthetic Data}

We generate a dictionary $\boldsymbol{D}$ of size $20 \times 50$, with each entry independently drawn from a normal distribution. Columns of $\boldsymbol{D}$ are then normalized to have unit norm. The training signals $\left\{\boldsymbol{y}_{l}\right\}_{l=1}^{L}$ are produced based on $\boldsymbol{D}$, where each signal $\boldsymbol{y}_{l}$ is a linear combination of $K_{l}$ randomly selected atoms and the weighting coefficients are i.i.d. normal random variables. Two different cases are considered. First, all training samples are generated with the same number of atoms, i.e. $K_{l}=K, \forall l$, and $K$ is assumed exactly known to the K-SVD method. The other case is that $K_{l}$ varies from 3 to 6 for different $l$ according to a uniform distribution. In this case, the K-SVD assumes that the sparsity level equals to 6 during the sparse coding stage. The observation noise is assumed multivariate Gaussian with zero mean and covariance matrix $\sigma^{2} \boldsymbol{I}$. Note that the APrU-DL (with FISTA) method requires to set two regularization parameters $\lambda$ and $\lambda_{s}$ to control the tradeoff between the sparsity and the data fitting error. The selection of these two parameters is always a tricky issue and an inappropriate 
choice may lead to considerable performance degradation. To show this, we use the following two different choices: $\left\{\lambda, \lambda_{s}\right\}=\{0.2,0.15\}$ and $\left\{\lambda, \lambda_{s}\right\}=\{0.4,0.4\}$, in which the former set of values are carefully selected by testing several pairs $\left\{\lambda, \lambda_{s}\right\}$ and choosing the best one, and the latter set of values slightly deviates from the former set of values. We use APrU-DL1 to denote the APrU-DL method which uses the former choice, and APrU-DL2 to denote the APrU-DL method which uses the latter one.

The recovery success rate is used to evaluate the dictionary learning performance. The success rate is computed as the ratio of the number of successfully recovered atoms to the total number of atoms. To calculate the success rate, the following steps are repeat $N$ times: 1) For the $i$ th atom $\boldsymbol{d}_{i}$, calculate the distance with all estimated atom, where the distance is defined as

$$
1-\frac{\left|\boldsymbol{d}_{i}^{T} \hat{\boldsymbol{d}}_{j}\right|}{\left\|\boldsymbol{d}_{i}\right\|\left\|\hat{\boldsymbol{d}}_{j}\right\|},
$$

where $\hat{\boldsymbol{d}}_{i}$ denotes the estimated atom. 2) Check whether there is an estimated atom having an distance smaller than 0.01 with it. If so, the considered the atom has been successfully recovered and remove the corresponding estimated atom. Table 1 shows the average recovery success rates of respective algorithms, where we set $L=1000$ and $L=2000$, respectively, and the signal-to-noise ratio (SNR) varies from 10 to 30dB. Results are averaged over 50 independent trials. From Table 1, we can see that:

- The proposed SBDL-Gibbs method achieves the highest recovery success rates in most cases. The proposed SBDL-VB method, although not as well as the SBDL-Gibbs, still provides quite comptetitive performance and presents a clear performance advantage over the K-SVD and APrU-DL methods when the number of training signals is limited, e.g. $L=1000$. In particular, both the SBDL-Gibbs and the SBDL-VB outperform the BPFA method by a big margin, despite all three methods were developed in a Bayesian framework.

- In the low SNR regime, e.g. SNR $=10 \mathrm{~dB}$, the K-SVD method suffers from a significant performance loss when there is a discrepancy between the presumed sparsity level and the groundtruth (see the case where $K_{l}$ varies but the presumed sparsity level is fixed to 6 ).

- The APrU-DL method is sensitive to the choice of the regularization parameters. It provides superior recovery performance when the regularization parameters are properly selected. Nevertheless, as we can see from Table 1, the APrU-DL method incurs a considerable performance degradation when the parameters deviate from their optimal choice, and there is no general guideline suggesting how to choose appropriate values for these regularization parameters.

To better illustrate the performance of those methods, we also evaluate them with three metric, namely, the achievable sparsity level (ASL), root mean square error (RMSE) and the average running 
time. The ASL is defined as

$$
\mathrm{ASL}=\frac{\mathrm{nnz}(\hat{\boldsymbol{X}})}{L}
$$

where $\operatorname{nnz}(\hat{\boldsymbol{X}})$ denotes the number of the nonzero entries of $\hat{\boldsymbol{X}}$ which denotes the sparse coding obtained by OMP. And RMSE is defined as

$$
\operatorname{RMSE}=\frac{\|\tilde{\boldsymbol{Y}}-\hat{\boldsymbol{D}} \hat{\boldsymbol{X}}\|_{F}}{\|\tilde{\boldsymbol{Y}}\|_{F}}
$$

where $\tilde{\boldsymbol{Y}}$ denotes the noise-free training samples and $\hat{\boldsymbol{D}}$ denotes learnt dictionary. Table 3 shows the ASL, RMSE and runtime results of the each algorithm with $L=1000$ and $K=4$. Form table 3, we see that the SBDL-Gibbs achieves best denoising performance. Concerning the ASL, we see that since the true sparsity level is known to the K-SVD method, it achieves the sparest representations in the cases of $\mathrm{SNR}=10 \mathrm{~dB}$ and $20 \mathrm{~dB}$. We also see that with the an increasing SNR, most methods can provide sparser representations and the proposed SBDL-Gibbs method even achieves a representation that sparser than the ground-truth in the case of $\mathrm{SNR}=30 \mathrm{~dB}$. Regarding the average runtime, since most algorithms are implemented with the fixed number of iterations, different SNRs don't influence their runtime. While for the SBDL-VB, we see that with the increasing of SNR, the algorithm needs fewer iterations to reach convergence. Additionally, we note that our proposed methods incur a higher complexity comparing with other methods, which is a major drawback of our proposed algorithms. This is because our proposed algorithms require conducting a matrix inverse operation when updating $\boldsymbol{X}$. The computational complexity required for this matrix inversion is of order $\mathcal{O}\left(N^{3}\right)$. To reduce the computational complexity of our methods, the approximate message passing (AMP) or generalized approximate message passing (GAMP) technique [33,34] may be employed for Bayesian inference and circumvent the matrix inverse operation. This is an important topic worthy of our future investigations. We also note that the lower computational complexity of APrU-DL compared with the proposed methods make it possible to select proper regularization parameters by trying different sets of parameters and selecting the best one.

\subsection{Application To Image Denoising}

We now demonstrate the results by applying the above methods to image denoising. Suppose images are corrupted by white Gaussian noise with zero mean and variance $\sigma^{2}$. We partition a noise-corrupted image into a number of overlapping patches (of size $8 \times 8$ pixels) obtained with one pixel shifting. Note that in our simulations, not all patches are selected for training, but only those patches whose top-left pixels are located at $[r \times i, r \times j]$ for any $i, j=0, \ldots,\lfloor(Q-8) / r\rfloor$ are selected, where $Q$ denotes the dimension of the $Q \times Q$ image, and $r$ is chosen to be $r=\{2,4\}$, respectively. The selected patches are then vectorized to generate the training signal $\left\{\boldsymbol{y}_{l}\right\}$. Also, 
in our experiments, we assume that the noise variance is perfectly known a priori by the K-SVD method. For the APrU-DL method, the regularization parameters $\lambda$ and $\lambda_{s}$ are carefully chosen to be $\lambda=25$ and $\lambda_{s}=30$. After the training by respective algorithms, the trained dictionary is then used for denoising. The denoising process involves a sparse coding of all patches (including those used for training and those not) of size $8 \times 8$ pixels from the noisy image which is performed using OMP. The final estimate of each pixel is obtained by averaging the associated pixel from each of the denoised overlapping patches in which this pixel is included.

We perform the algorithms with three benchmark images, namely, "boat" $(256 \times 256)$, "cameraman" $(256 \times 256)$, and "couple" $(512 \times 512)$. Table 2 shows the peak signal to noise ratio (PSNR) results obtained for different nature images by respective algorithms, where the noise standard deviation is set to $\sigma=\{15,25,50\}$, respectively, and the dictionary to be inferred is assumed of size $64 \times 256$. The PSNR is defined as

$$
\operatorname{PSNR}=20 \log _{10}\left(\frac{255 \times Q^{2}}{\|\hat{\boldsymbol{U}}-\boldsymbol{U}\|_{F}}\right),
$$

where $\hat{\boldsymbol{U}}$ and $\boldsymbol{U}$ denote the denoised image and the original noise-free image, respectively. From Table 2, we see that the results of all methods are very close to each other in general. The proposed SBDL-Gibbs achieves a slightly higher PSNR than other methods in most cases, particularly when fewer number of signals is used for training. This result again demonstrates the superiority of the proposed method. The denoising performance for $r=2$ cases are also evaluated by two metrics, namely, structural similarity (SSIM) index [35] and ASL. The results are shown in table 4. Form table 4, we see that all the algorithms provide similar achievable sparsity levels and SSIM indexes. The proposed SBDL-Gibbs achieves a slightly higher SSIM than other methods in most cases. The achievable sparsity level of SBDL-Gibbs is similar to other methods. In Fig. 2, 3, 4, 5 and 6, we present the noise-corrupted images "cameraman" and "couple", and the denoised images using dictionaries trained by respective algorithms. To better show the difference of the denoised image, we zoom in a part of the denoised images in Fig. 4, 5 and 6 and show them in Fig. 7. The zoomed in part of the images which is of size $100 \times 100$ locates at the top left corner of door in the images. Fig. 8 plots the dictionaries learnt by the methods from the image "couple" with $\sigma=50$ and $r=2$. From Fig. 7 and 8, we see that the APrU-DL and SBDL-VB methods provide noisy dictionaries as well as slightly dim denoised images comparing with other methods.

We note that the proposed SBDL-Gibbs achieves better image denoising performance than the BPFA method. The BPFA is a state-of-the-art method which provides superior performance for image denoising, as illustrated by many experiments. Nevertheless, in [11], the BPFA uses all patches of a test image, based on which the clustering effects of adjacent patches are utilized to improve the performance. In our experiments, we, following [4], only use a subset of all patches, 
instead of all patches, for dictionary learning. Specifically, one half (corresponding to $r=2$ ) and one fourth (corresponding to $r=4$ ) of all patches are used. Since only a portion of patches are used for training, the benefit brought by the clustering effect may be limited, which possibly, is the reason why the BPFA method is not as good as our proposed method.

\section{Conclusions}

We developed a new Bayesian hierarchical model for learning overcomplete dictionaries based on a set of training data. This new framework extends the conventional sparse Beysian learning framework to deal with the dictionary learning problem. Specifically, a Gaussian-inverse Gamma hierarchical prior is used to promote the sparsity of the representation. Suitable priors are also placed on the dictionary and the noise variance such that they can be inferred from the data. We developed a variational Bayesian method and a Gibbs sampler for Bayesian inference. Unlike some of previous methods, the proposed methods do not need to assume knowledge of the noise variance a priori, and can infer the noise variance automatically from the data. The performance of the proposed methods is evaluated using synthetic data. Numerical results show that the proposed methods are able to learn the dictionary with an accuracy notably better than the existing methods, particularly for the case where there is a limited number of training signals. The proposed methods are also applied to image denoising, where superior denoising results are achieved even compared with other state-ofthe-art algorithms. Our proposed hierarchical model is also flexible to incorporate additional prior information to enhance the dictionary learning performance.

\section{References}

[1] E. Candés and T. Tao, "Decoding by linear programming," IEEE Trans. Information Theory, no. 12, pp. 4203-4215, Dec. 2005.

[2] J. M. Duarte-Carvajalino and G. Sapiro, "Learning to sense sparse signals: simultaneous sensing matrix and sparsifying dictionary optimization," IEEE Trans. Image Processing, vol. 18, no. 7, pp. 1395-1408, Jul. 2009.

[3] J. Wright, A. Y. Yang, A. Ganesh, S. S. Sastry, and Y. Ma, "Robust face recognition vis sparse representation," IEEE Trans. Pattern Analysis and Machine Intelligence, vol. 31, no. 2, pp. 210-227, Feb. 2009.

[4] M. Aharon, M. Elad, and A. Bruckstein, "K-SVD: an algorithm for designing overcomplete dictionaries for sparse representation," IEEE Trans. Signal Processing, vol. 54, no. 11, pp. 4311-4322, Nov. 2006. 
[5] M. Elad and M. Aharon, "Image denoising via sparse and redundant representations over learned dictionaries," IEEE Trans. Image Processing, vol. 15, no. 12, pp. 3736-3745, Dec. 2006.

[6] J. Mairal, F. Bach, and J. Ponce, "Task-driven dictionary learning," IEEE Trans. Pattern Analysis and Machine Intelligence, vol. 34, no. 4, pp. 791-804, Apr. 2012.

[7] K. Engan, S. O. Aase, and J. H. Hakon-Husoy, "Method of optimal directions for frame design," in IEEE International Conference on Acoustics, Speech and Signal Processing, Phoenix, AZ, March 15-19 1999.

[8] M. Yaghoobi, T. Blumensath, and M. E. Davies, "Dictionary learning for sparse approximations with the majorization method," IEEE Trans. Signal Processing, vol. 57, no. 6, pp. 2178-2191, Jun. 2009.

[9] W. Dai, T. Xu, and W. Wang, "Simultaneous codeword optimization (SimCO) for dictionary update and learning," IEEE Trans. Signal Processing, vol. 60, no. 12, pp. 6340-6353, Dec. 2012.

[10] M. Sadeghi, M. Babaie-Zadeh, and C. Jutten, "Learning overcomplete dictionaries based on atom-by-atom updating," IEEE Trans. Signal Processing, vol. 62, no. 4, pp. 883-891, Feb. 2014.

[11] M. Zhou, H. Chen, J. Paisley, L. Ren, L. Li, Z. Xing, D. Dunson, G. Sapiro, and L. Carin, "Nonparametric Bayesian dictionary learning for analysis of noisy and incomplete images," IEEE Trans. Image Processing, vol. 21, no. 1, pp. 130-144, Jan. 2012.

[12] J. Mairal, F. Bach, J. Ponce, and G. Sapiro, "Online learning for matrix factorization and sparse coding," Journal of Machine Learning Research, vol. 11, pp. 19-60, 2010.

[13] K. Skretting and K. Engan, "Recursive least squares dictionary learning algorithm," IEEE Trans. Signal Processing, vol. 58, no. 4, pp. 2121-2130, Apr. 2010.

[14] K. Labusch, E. Barth, and T. Martinetz, "Robust and fast learning of sparse codes with stochastic gradient descent," IEEE Journal of Selected Topics in Signal Processing, vol. 5, no. 5, pp. 1048-1060, 2011.

[15] A. Koppel, G. Warnell, E. Stump, and A. Ribeiro, "D4l: Decentralized dynamic discriminative dictionary learning," in 2015 IEEE/RSJ International Conference on Intelligent Robots and Systems (IROS). IEEE, 2015, pp. 2966-2973.

[16] H. Raja and W. U. Bajwa, "Cloud K-SVD: A collaborative dictionary learning algorithm for big, distributed data," IEEE Transactions on Signal Processing, vol. 64, no. 1, pp. 173-188, Jan. 2016. 
[17] M. Tipping, "Sparse Bayesian learning and the relevance vector machine," Journal of Machine Learning Research, vol. 1, pp. 211-244, 2001.

[18] Z. Zhang, S. Wang, D. Liu, and M. I. Jordan, "EP-GIG priors and applications in Bayesian sparse learning," The Journal of Machine Learning Research, vol. 13, no. 1, pp. 2031-2061, 2012.

[19] D. G. Tzikas, A. C. Likas, and N. P. Galatsanos, "The variational approximation for Bayesian inference," IEEE Signal Processing Magazine, pp. 131-146, Nov. 2008.

[20] R. E. Turner and M. Sahani, "Two problems with variational expectation maximisation for time-series models," in Workshop on Inference and Estimation in Probabilistic Time-Series Models, vol. 2, no. 3, 2008.

[21] T. S. Jaakkola and Y. Qi, "Parameter expanded variational bayesian methods," in Advances in Neural Information Processing Systems, 2006, pp. 1097-1104.

[22] C. M. Bishop, Pattern recognition and machine learning. Springer, 2007.

[23] D. P. Wipf and B. D. Rao, "An empirical Bayesian strategy for solving the simultaneous sparse approximation problem," IEEE Trans. Signal Processing, vol. 55, no. 7, pp. 3704-3716, Jul. 2007.

[24] S. Ji, Y. Xue, and L. Carin, "Bayesian compressive sensing," IEEE Trans. Signal Processing, vol. 56, no. 6, pp. 2346-2356, Jun. 2008.

[25] L. Wang, L. Zhao, G. Bi, and C. Wan, "Hierarchical sparse signal recovery by variational Bayesian inference," IEEE Signal Processing Letters, vol. 21, no. 1, pp. 110-113, 2014.

[26] L. Zhao, G. Bi, L. Wang, and H. Zhang, "An improved auto-calibration algorithm based on sparse Bayesian learning framework," IEEE Signal Processing Letters, vol. 20, no. 9, pp. 889$892,2013$.

[27] Y. Guan and J. G. Dy, "Sparse probabilistic principal component analysis," in International Conference on Artificial Intelligence and Statistics, 2009, pp. 185-192.

[28] J. Fang, Y. Shen, H. Li, and P. Wang, "Pattern-coupled sparse Bayesian learning for recovery of block-sparse signals," IEEE Trans. Signal Processing, vol. 63, no. 2, pp. 360-372, Jan. 2015.

[29] S. Kullback and R. A. Leibler, "On information and sufficiency," The annals of mathematical statistics, pp. 79-86, 1951.

[30] K. P. Murphy, Machine learning: a probabilistic perspective. MIT press, 2012. 
[31] A. Gelman, J. B. Carlin, H. S. Stern, D. B. Dunson, A. Vehtari, and D. B. Rubin, Bayesian data analysis. Chapman and Hall/CRC, third edition, 2013.

[32] B. Walsh, "Markov chain monte carlo and gibbs sampling," 2004.

[33] D. L. Donoho, A. Maleki, and A. Montanari, "Message passing algorithms for compressed sensing: I. motivation and construction," in Proc. Inf. Theory Workshop, Cairo, Egypt, Jan. 2010.

[34] S. Rangan, "Generalized approximate message passing for estimation with random linear mixing," in Proc. IEEE Int. Symp. Inf. Theory (ISIT), Saint Petersburg, Russia, Aug. 2011.

[35] Z. Wang, A. C. Bovik, H. R. Sheikh, and E. P. Simoncelli, "Image quality assessment: from error visibility to structural similarity," IEEE Transactions on Image Processing, vol. 13, no. 4, pp. 600-612, 2004. 

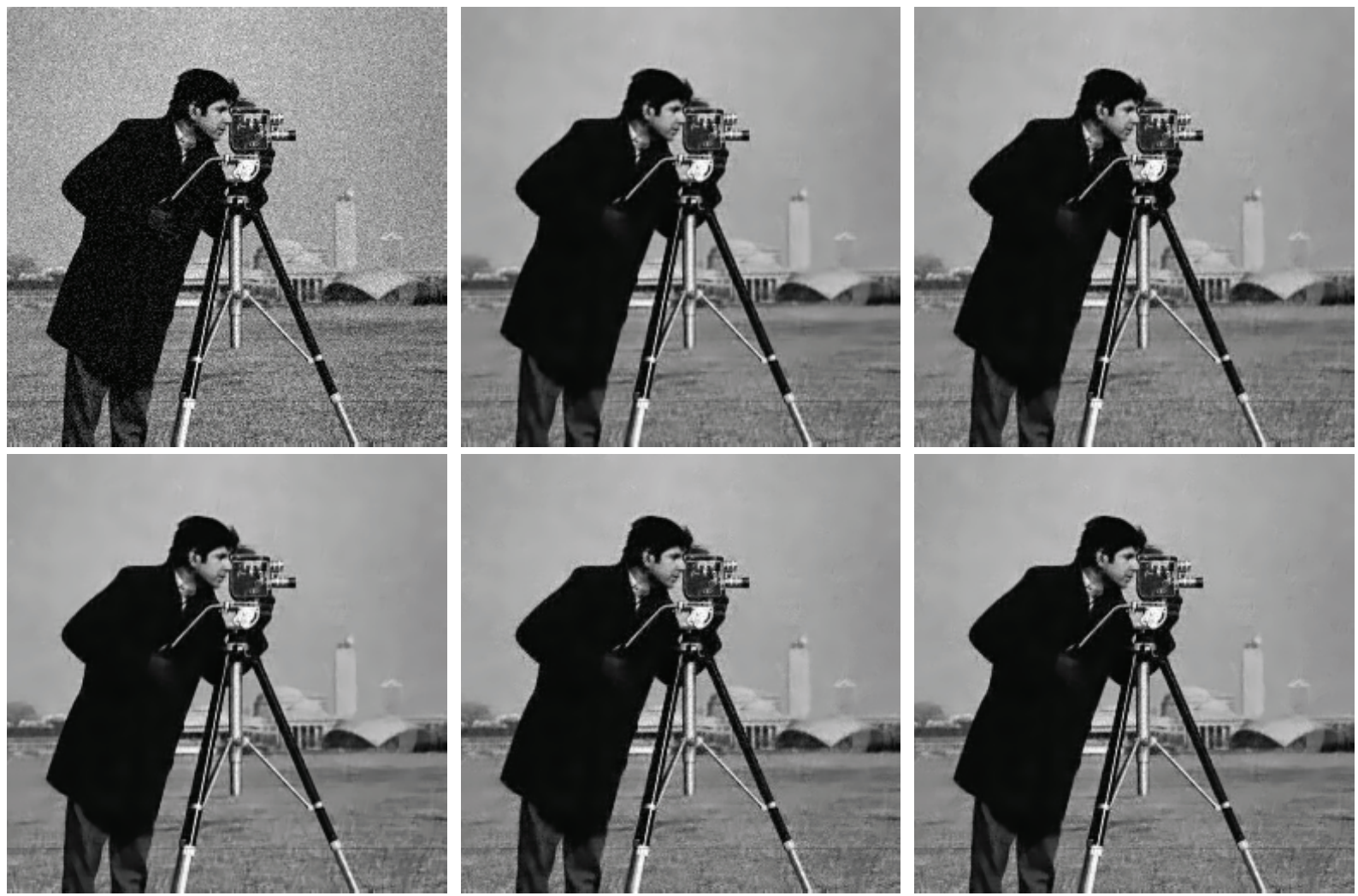

Figure 2: Denoising results of each method $(\sigma=15$ and $r=4)$ for image "cameraman". From left to right: the corrupted image, the denoised image by KSVD (PSNR=31.3553dB,SSIM=0.8938), BPFA $(P S N R=31.1063 d B, S S I M=0.8906), \quad A P r U-D L(P S N R=31.5541 d B, S S I M=0.8952), \quad$ SBDL-VB $(\mathrm{PSNR}=31.0739 \mathrm{~dB}, \mathrm{SSIM}=0.8913), \mathrm{SBDL}-\mathrm{Gibbs}(\mathrm{PSNR}=31.4931 \mathrm{~dB}, \mathrm{SSIM}=0.8968)$. 
Table 1: Recovery Success Rates

\begin{tabular}{|c|c|c|c|c|c|c|}
\hline$L$ & SNR & Algorithm & $K=3$ & $K=4$ & $K=5$ & Var. $K$ \\
\hline \multirow{18}{*}{1000} & \multirow{6}{*}{10} & K-SVD & 80.52 & 36.36 & 2.52 & 0.80 \\
\hline & & BPFA & 76.76 & 57.12 & 22.56 & 43.32 \\
\hline & & APrU-DL1 & 85.64 & 64.40 & 33.44 & 53.68 \\
\hline & & APrU-DL2 & 48.20 & 17.48 & 4.68 & 12.52 \\
\hline & & SBDL-VB & 86.00 & 63.84 & 16.28 & 47.48 \\
\hline & & SBDL-Gibbs & 91.52 & 62.48 & 6.32 & 41.80 \\
\hline & \multirow{6}{*}{20} & K-SVD & 93.20 & 93.44 & 92.08 & 84.68 \\
\hline & & BPFA & 87.96 & 92.00 & 94.08 & 93.58 \\
\hline & & APrU-DL1 & 94.04 & 93.32 & 87.76 & 93.48 \\
\hline & & APrU-DL2 & 72.48 & 40.32 & 14.15 & 33.04 \\
\hline & & SBDL-VB & 97.28 & 95.96 & 92.32 & 94.48 \\
\hline & & SBDL-Gibbs & 99.64 & 99.16 & 97.52 & 99.12 \\
\hline & \multirow{6}{*}{30} & K-SVD & 94.24 & 94.32 & 93.92 & 86.64 \\
\hline & & BPFA & 87.04 & 91.20 & 94.56 & 92.60 \\
\hline & & APrU-DL1 & 94.24 & 94.92 & 88.16 & 93.96 \\
\hline & & APrU-DL2 & 73.40 & 43.16 & 17.16 & 34.36 \\
\hline & & SBDL-VB & 96.60 & 96.16 & 92.32 & 95.48 \\
\hline & & SBDL-Gibbs & 99.60 & 99.16 & 98.64 & 99.00 \\
\hline \multirow{18}{*}{2000} & \multirow{6}{*}{10} & K-SVD & 91.00 & 88.88 & 50.56 & 25.32 \\
\hline & & BPFA & 91.16 & 92.28 & 86.44 & 90.84 \\
\hline & & APrU-DL1 & 97.00 & 94.88 & 86.24 & 95.44 \\
\hline & & APrU-DL2 & 84.84 & 68.36 & 42.28 & 64.04 \\
\hline & & SBDL-VB & 92.92 & 81.80 & 55.68 & 77.16 \\
\hline & & SBDL-Gibbs & 98.56 & 95.72 & 80.20 & 93.88 \\
\hline & \multirow{6}{*}{20} & K-SVD & 95.64 & 96.68 & 95.16 & 94.00 \\
\hline & & BPFA & 89.68 & 91.76 & 94.72 & 93.04 \\
\hline & & APrU-DL1 & 95.40 & 96.48 & 95.80 & 96.56 \\
\hline & & APrU-DL2 & 85.32 & 82.44 & 64.48 & 79.84 \\
\hline & & SBDL-VB & 97.64 & 96.56 & 92.12 & 95.04 \\
\hline & & SBDL-Gibbs & 99.48 & 99.56 & 98.92 & 99.16 \\
\hline & \multirow{6}{*}{30} & K-SVD & 95.88 & 96.92 & 96.96 & 93.36 \\
\hline & & BPFA & 87.24 & 91.80 & 95.92 & 93.94 \\
\hline & & APrU-DL1 & 94.28 & 95.00 & 96.80 & 95.64 \\
\hline & & APrU-DL2 & 86.32 & 82.40 & 66.08 & 80.52 \\
\hline & & SBDL-VB & 96.88 & 96.96 & 92.96 & 94.96 \\
\hline & & SBDL-Gibbs & 99.40 & 99.16 & 99.52 & 99.32 \\
\hline
\end{tabular}


Table 2: PSNR Results

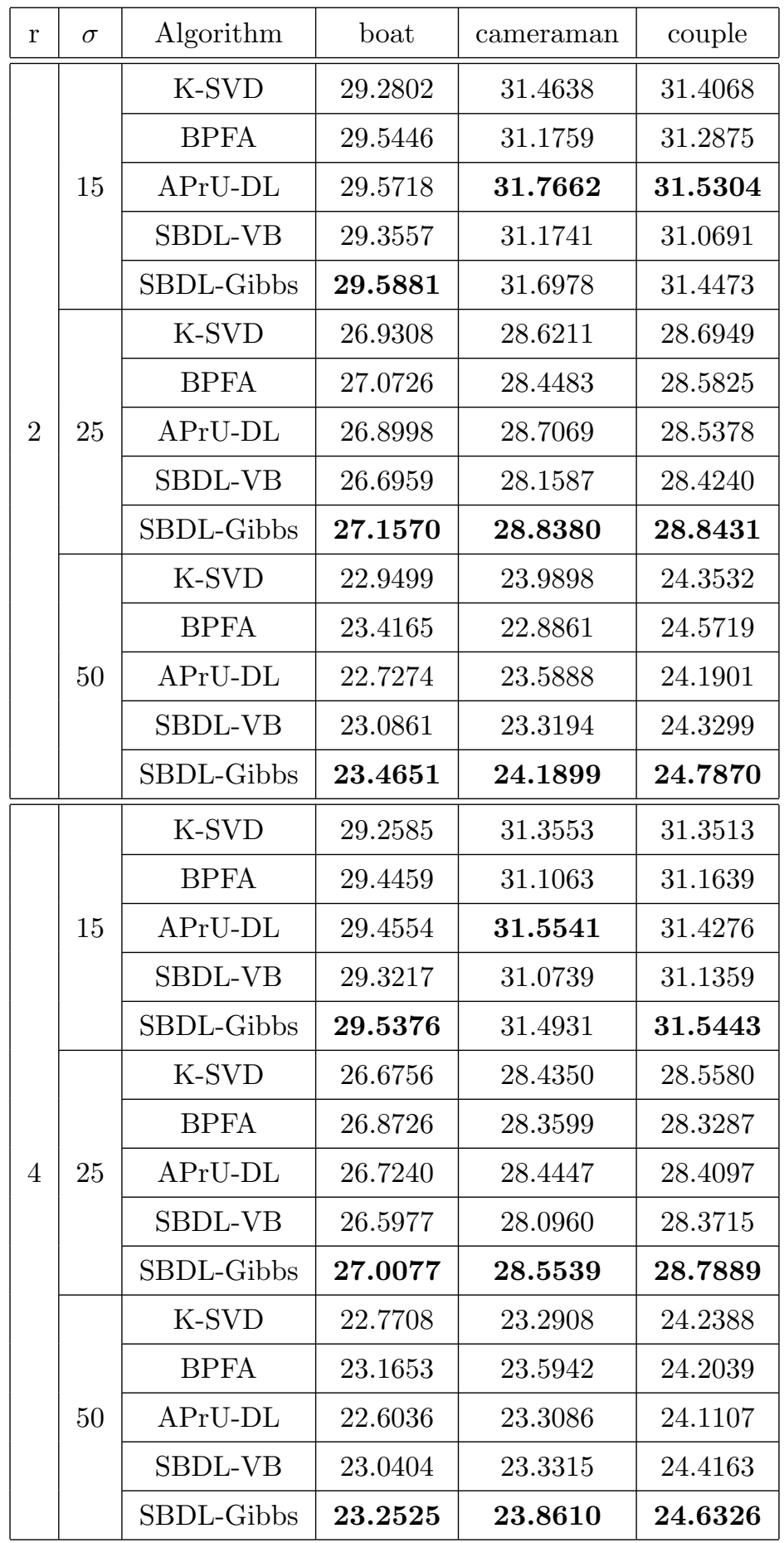


Table 3: Results of ASL, RMSE and Running Time

\begin{tabular}{|c|c|c|c|c|}
\hline $\operatorname{SNR}(\mathrm{dB})$ & Algorithm & ASL & RMSE & runtime \\
\hline \multirow{6}{*}{10} & K-SVD & 4 & 0.3064 & 0.9778 \\
\hline & BPFA & 9.4243 & 0.3092 & 27.3396 \\
\hline & APrU-DL1 & 9.2787 & 0.3086 & 19.1625 \\
\hline & APrU-DL2 & 15.5645 & 0.3113 & 12.1774 \\
\hline & SBDL-VB & 9.1543 & 0.3090 & 91.6423 \\
\hline & SBDL-Gibbs & 9.3625 & 0.3086 & 154.9286 \\
\hline \multirow{6}{*}{20} & K-SVD & 4 & 0.1344 & 0.9563 \\
\hline & BPFA & 5.1772 & 0.0862 & 29.3492 \\
\hline & APrU-DL1 & 5.2479 & 0.0975 & 18.4018 \\
\hline & APrU-DL2 & 5.7878 & 0.1158 & 11.6770 \\
\hline & SBDL-VB & 4.6019 & 0.0792 & 55.6192 \\
\hline & SBDL-Gibbs & 4.3463 & 0.0766 & 154.6508 \\
\hline \multirow{6}{*}{30} & K-SVD & 4 & 0.1127 & 0.9757 \\
\hline & BPFA & 5.7770 & 0.0683 & 33.6997 \\
\hline & APrU-DL1 & 4.3184 & 0.0772 & 18.4779 \\
\hline & APrU-DL2 & 4.5205 & 0.0904 & 11.7725 \\
\hline & SBDL-VB & 4.0543 & 0.577 & 58.5767 \\
\hline & SBDL-Gibbs & 3.8007 & 0.0536 & 155.2510 \\
\hline
\end{tabular}


Table 4: SSIM/ASL Results

\begin{tabular}{|c|c|c|c|c|c|}
\hline $\mathrm{r}$ & $\sigma$ & Algorithm & boat & cameraman & couple \\
\hline \multirow{15}{*}{2} & \multirow{5}{*}{15} & K-SVD & $0.8233 / 4.06$ & $0.8959 / 2.50$ & $0.8517 / 1.93$ \\
\hline & & BPFA & $0.8345 / 4.72$ & $0.8911 / 4.12$ & $0.8528 / 3.55$ \\
\hline & & APrU-DL & $0.8267 / 3.68$ & $0.8975 / 2.30$ & $0.8529 / 1.98$ \\
\hline & & SBDL-VB & $0.8300 / 4.89$ & $0.8922 / 3.53$ & $0.8457 / 2.77$ \\
\hline & & SBDL-Gibbs & $\mathbf{0 . 8 3 7 7} / 4.01$ & $\mathbf{0 . 8 9 9 0} / 2.45$ & $0.8579 / 2.18$ \\
\hline & \multirow{5}{*}{25} & K-SVD & $0.7313 / 1.81$ & $0.8332 / 1.16$ & $0.7710 / 0.94$ \\
\hline & & BPFA & $0.7497 / 2.23$ & $0.8295 / 1.90$ & $0.7735 / 1.74$ \\
\hline & & APrU-DL & $0.7299 / 1.83$ & $0.8312 / 1.21$ & $0.7640 / 1.04$ \\
\hline & & SBDL-VB & $0.7357 / 2.44$ & $0.8244 / 1.84$ & $0.7641 / 1.30$ \\
\hline & & SBDL-Gibbs & $\mathbf{0 . 7 5 3 3} / 1.80$ & $0.8374 / 1.18$ & $\mathbf{0 . 7 8 1 3} / 1.01$ \\
\hline & \multirow{5}{*}{50} & K-SVD & $0.5540 / 0.39$ & $0.7110 / 0.30$ & $0.5918 / 0.23$ \\
\hline & & BPFA & $0.5817 / 0.48$ & $0.7067 / 0.42$ & $0.6062 / 0.34$ \\
\hline & & APrU-DL & $0.5438 / 0.44$ & $0.7010 / 0.36$ & $0.5841 / 0.24$ \\
\hline & & SBDL-VB & $0.5649 / 0.50$ & $0.6928 / 0.48$ & $0.5906 / 0.28$ \\
\hline & & SBDL-Gibbs & $\mathbf{0 . 5 8 4 5} / 0.40$ & $\mathbf{0 . 7 1 6 7} / 0.32$ & $\mathbf{0 . 6 1 7 0} / 0.23$ \\
\hline
\end{tabular}



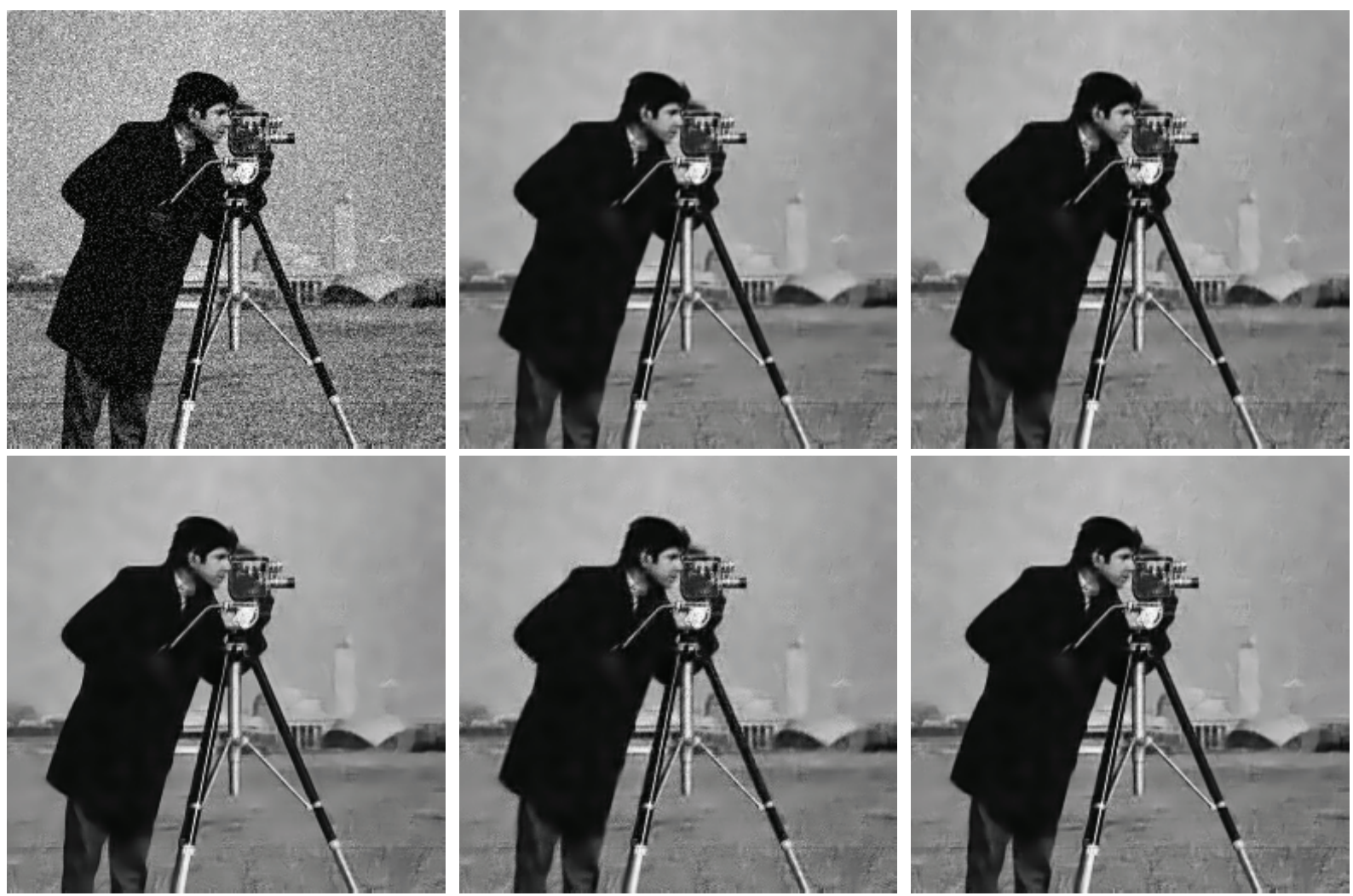

Figure 3: Denoising results of each method ( $\sigma=25$ and $r=2)$ for image "cameraman". From left to right: the corrupted image, the denoised image by KSVD (PSNR=28.6211dB,SSIM=0.8332), BPFA(PSNR=28.4483dB,SSIM=0.8295), APrU-DL $(\mathrm{PSNR}=28.7069 \mathrm{~dB}, \mathrm{SSIM}=0.8312), \quad$ SBDL-VB $(\mathrm{PSNR}=28.1587 \mathrm{~dB}, \mathrm{SSIM}=0.8244), \mathrm{SBDL}-\mathrm{Gibbs}(\mathrm{PSNR}=28.8380 \mathrm{~dB}, \mathrm{SSIM}=0.8374)$. 

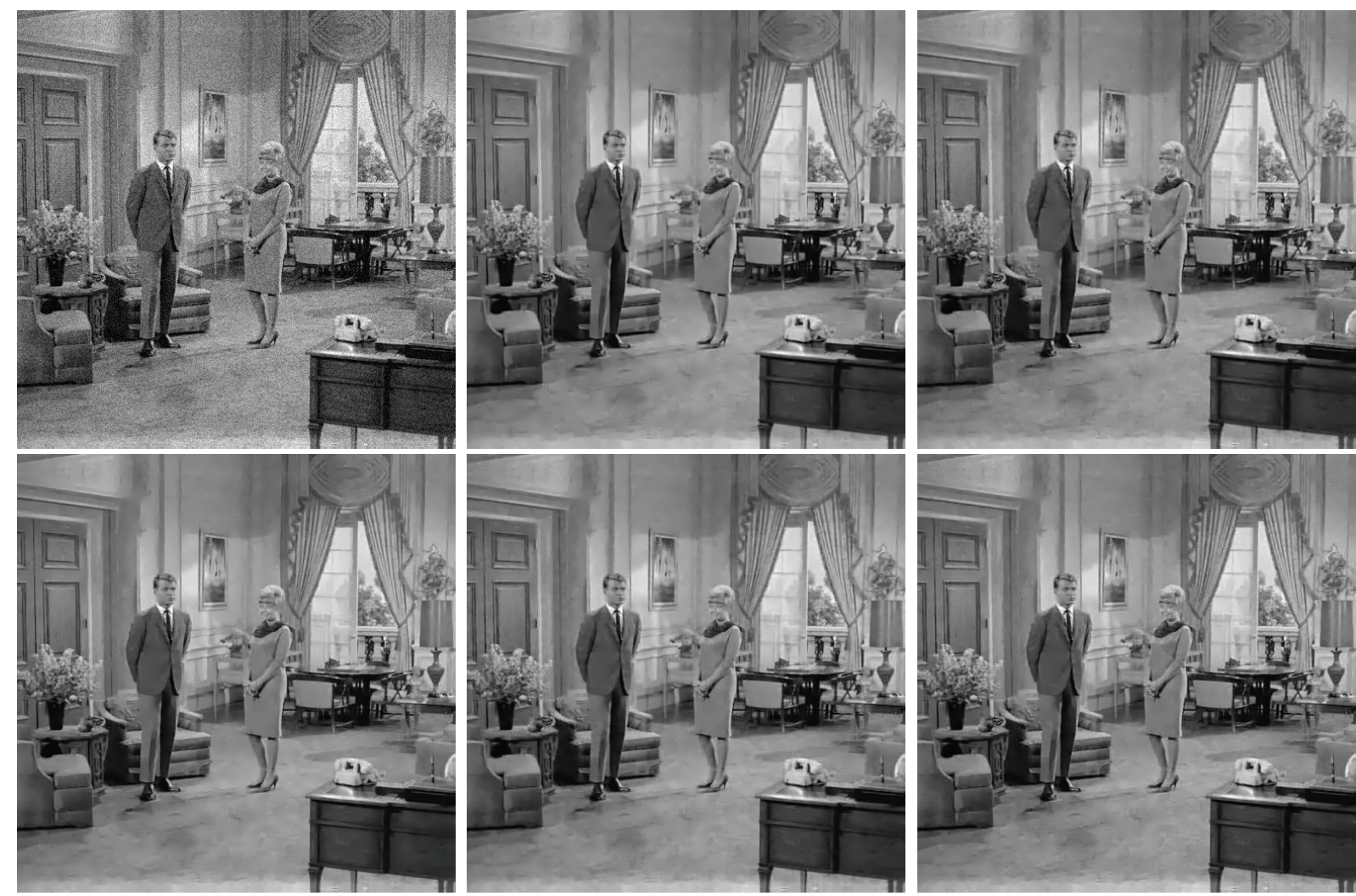

Figure 4: Denoising results of each method $(\sigma=15$ and $r=4)$ for image "couple". From left to right: the corrupted image, the denoised image by KSVD (PSNR=31.3513dB,SSIM=0.8504), BPFA $(\mathrm{PSNR}=31.1639 \mathrm{~dB}, \mathrm{SSIM}=0.8481), \quad \mathrm{APr} U-\mathrm{DL}(\mathrm{PSNR}=31.4276 \mathrm{~dB}, \mathrm{SSIM}=0.8511), \quad$ SBDL-VB $(\mathrm{PSNR}=31.1359 \mathrm{~dB}, \mathrm{SSIM}=0.8482), \mathrm{SBDL}-\mathrm{Gibbs}(\mathrm{PSNR}=31.5443 \mathrm{~dB}, \mathrm{SSIM}=0.8584)$. 

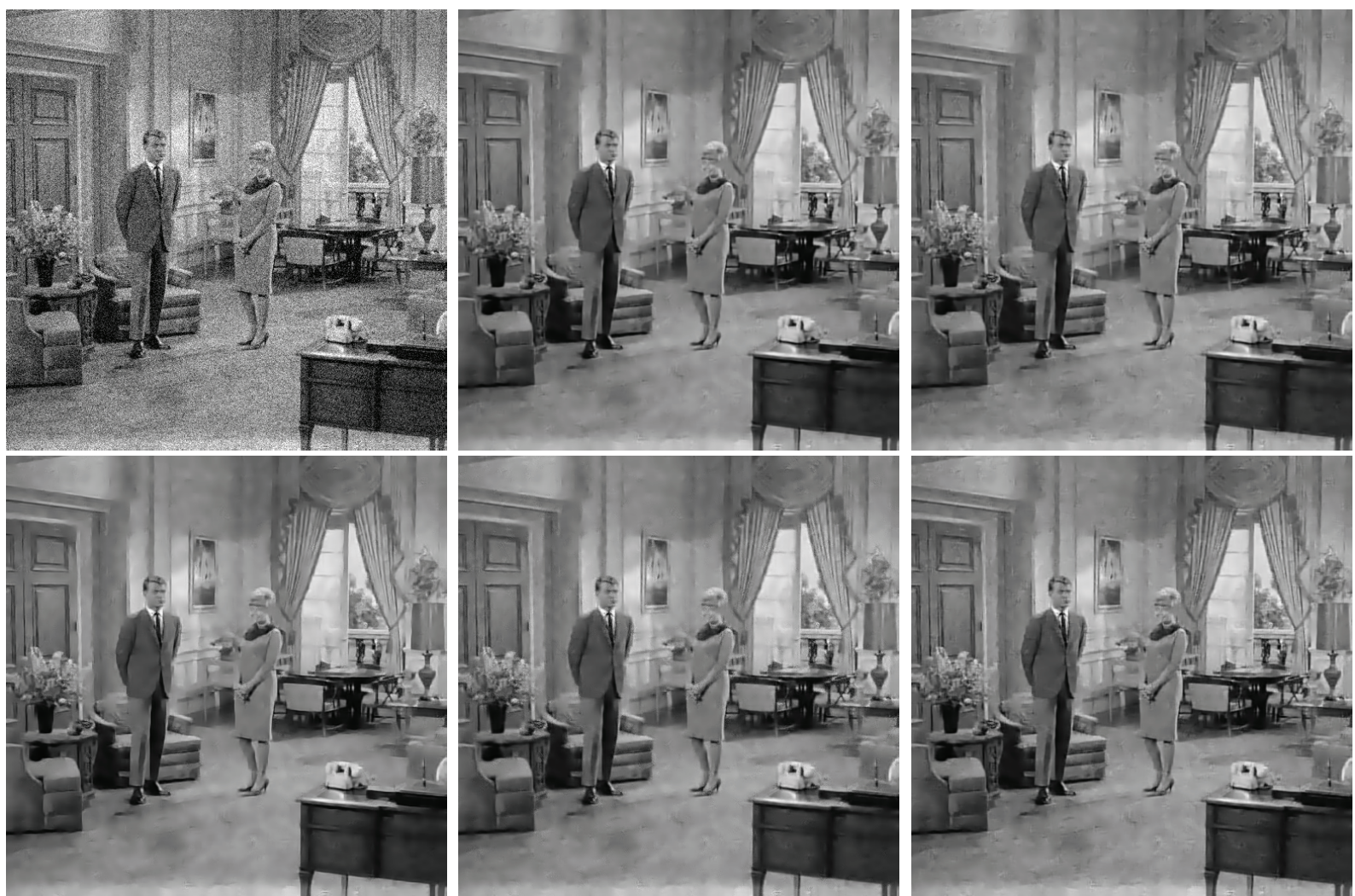

Figure 5: Denoising results of each method $(\sigma=25$ and $r=2)$ for image "couple". From left to right: the corrupted image, the denoised image by KSVD (PSNR=28.6949dB,SSIM=0.7710), BPFA(PSNR=28.5825dB,SSIM=0.7735), APrU-DL(PSNR=28.5378dB,SSIM=0.7640), SBDL-VB $(\mathrm{PSNR}=28.4240 \mathrm{~dB}, \mathrm{SSIM}=0.7641), \mathrm{SBDL}-\mathrm{Gibbs}(\mathrm{PSNR}=28.8431 \mathrm{~dB}, \mathrm{SSIM}=0.7813)$. 

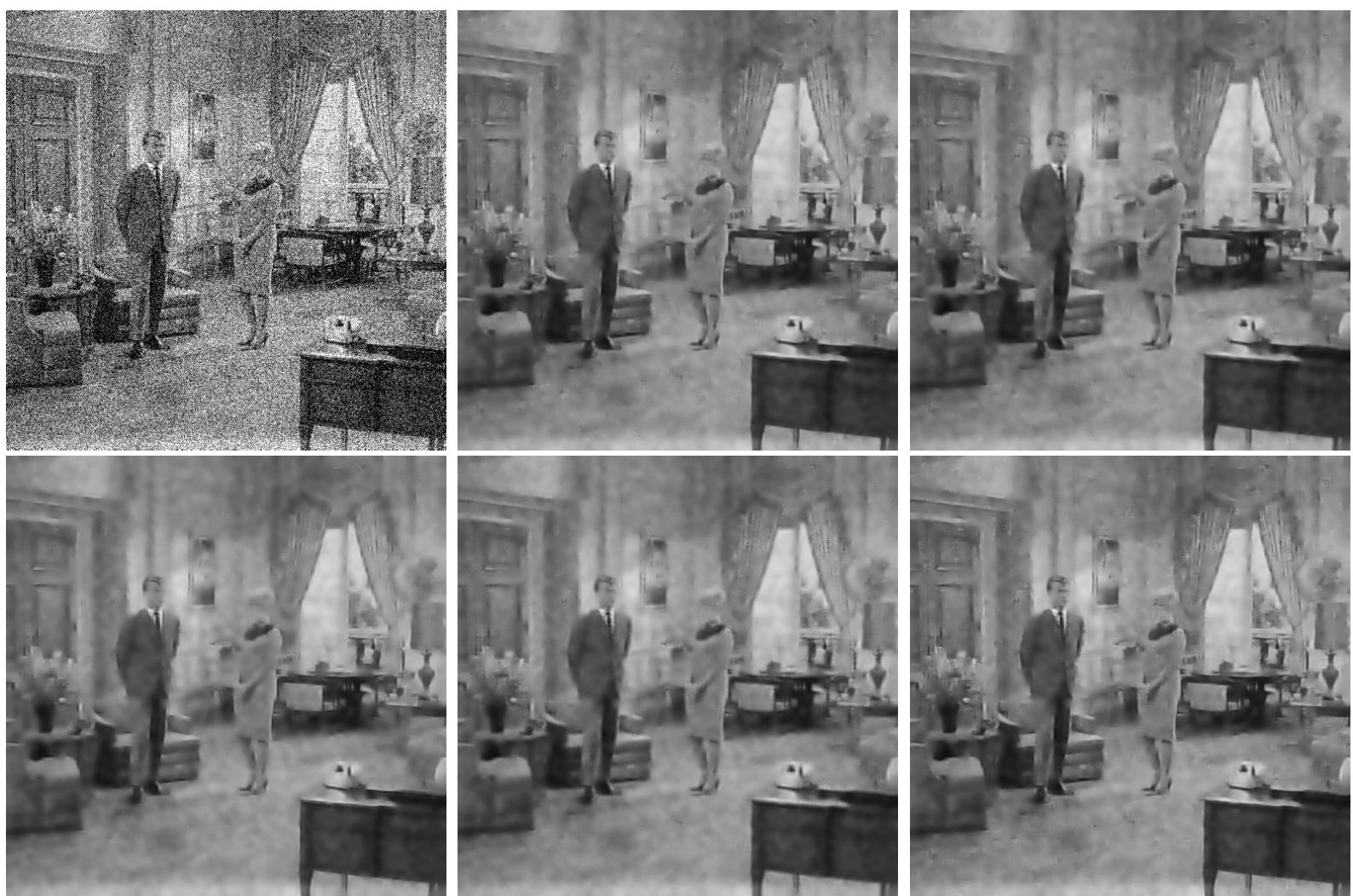

Figure 6: Denoising results of each method $(\sigma=50$ and $r=2)$ for image "couple". From left to right: the corrupted image, the denoised image by KSVD (PSNR=24.3532dB,SSIM=0.5918), BPFA(PSNR=24.5719dB,SSIM=0.6062), APrU-DL(PSNR=24.1901dB,SSIM=0.5841), SBDL-VB $(\mathrm{PSNR}=24.3299 \mathrm{~dB}, \mathrm{SSIM}=0.5906), \mathrm{SBDL}-\mathrm{Gibbs}(\mathrm{PSNR}=24.7870 \mathrm{~dB}, \mathrm{SSIM}=0.6170)$. 

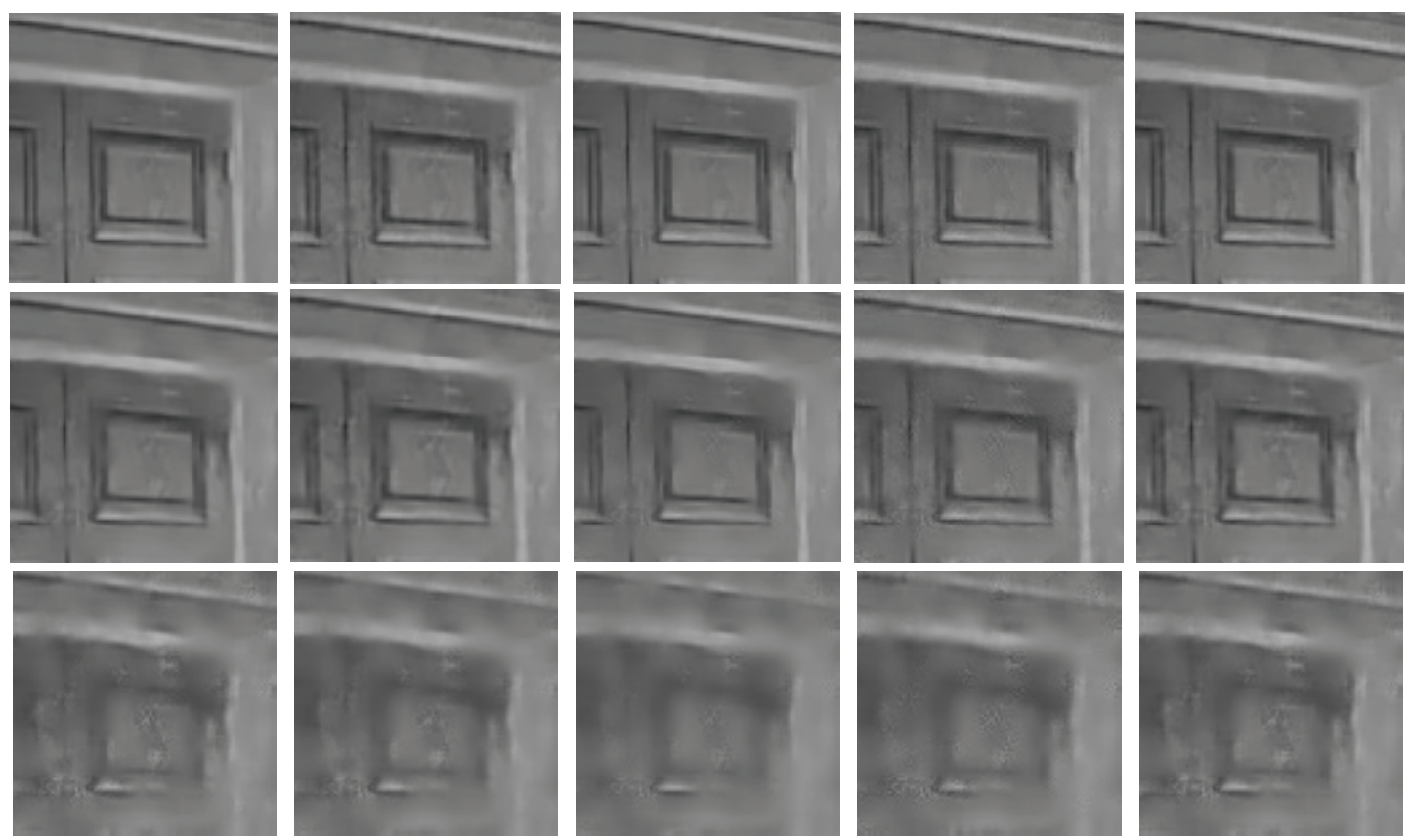

Figure 7: A part of zoomed in images denoised by each method for image "couple". From left to right: zoom in of the image denoised by KSVD, BPFA, APrU-DL, SBDL-VB, SBDL-Gibbs. Top row: $\sigma=15$ and $r=4$. Middle row: $\sigma=25$ and $r=2$. Bottom row: $\sigma=50$ and $r=2$. 

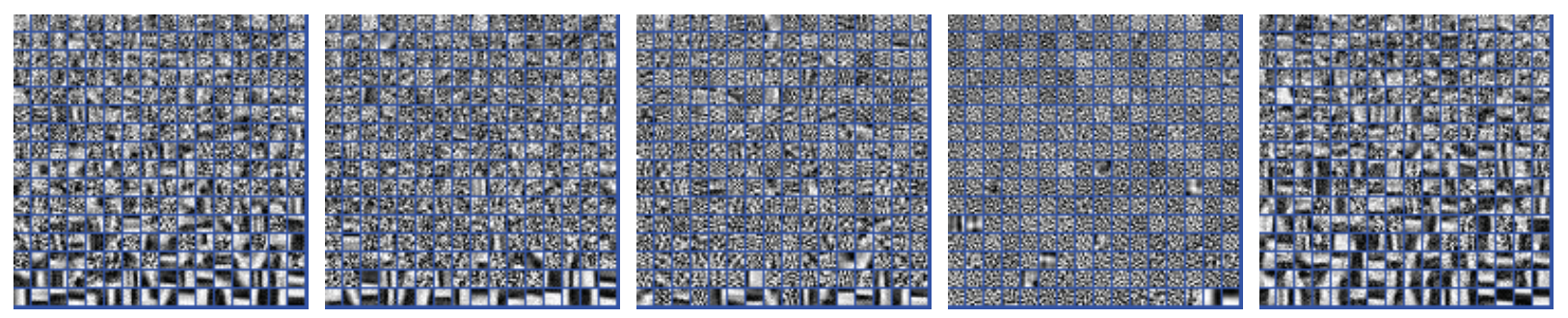

Figure 8: Dictionaries leant by each method $(\sigma=50$ and $r=2)$ for image "couple". From left to right: the dictionary learnt by KSVD, BPFA, APrU-DL, SBDL-VB, SBDL-Gibbs. 